\title{
The early star generations: the dominant effect of rotation on the CNO yields
}

\author{
G. Meynet, S. Ekström, and A. Maeder
}

Geneva Observatory, 1290 Sauverny, Switzerland

e-mail: georges.meynet@obs.unige.ch

Received 15 March 2005 / Accepted 27 September 2005

\begin{abstract}
Aims. We examine the role of rotation on the evolution and chemical yields of very metal-poor stars.

Methods. The models include the same physics, which was applied successfully at the solar $Z$ and for the SMC, in particular, shear diffusion, meridional circulation, horizontal turbulence, and rotationally enhanced mass loss.

Results. Models of very low $Z$ experience a much stronger internal mixing in all phases than at solar $Z$. Also, rotating models at very low $Z$, contrary to the usual considerations, show a large mass loss, which mainly results from the efficient mixing of the products of the $3 \alpha$ reaction into the H-burning shell. This mixing allows convective dredge-up to enrich the stellar surface in heavy elements during the red supergiant phase, which in turn favours a large loss of mass by stellar winds, especially as rotation also increases the duration of this phase. On the whole, the low $Z$ stars may lose about half of their mass. Massive stars initially rotating at half of their critical velocity are likely to avoid the pair-instability supernova. The chemical composition of the rotationally enhanced winds of very low $Z$ stars show large $\mathrm{CNO}$ enhancements by factors of $10^{3}$ to $10^{7}$, together with large excesses of ${ }^{13} \mathrm{C}$ and ${ }^{17} \mathrm{O}$ and moderate amounts of $\mathrm{Na}$ and $\mathrm{Al}$. The excesses of primary $\mathrm{N}$ are particularly striking. When these ejecta from the rotationally enhanced winds are diluted with the supernova ejecta from the corresponding $\mathrm{CO}$ cores, we find $[\mathrm{C} / \mathrm{Fe}],[\mathrm{N} / \mathrm{Fe}],[\mathrm{O} / \mathrm{Fe}]$ abundance ratios that are very similar to those observed in the $\mathrm{C}$-rich, extremely metal-poor stars (CEMP). We show that rotating AGB stars and rotating massive stars have about the same effects on the CNO enhancements. Abundances of s-process elements and the ${ }^{12} \mathrm{C} /{ }^{13} \mathrm{C}$ ratio could help us to distinguish between contributions from AGB and massive stars.
\end{abstract}

Key words. stars: evolution - stars: rotation - stars: abundances - stars: mass loss - nuclear reactions, nucleosynthesis, abundances

\section{Introduction}

Interest in the evolution of extremely metal-poor stars has been stimulated recently by at least two types of observing programmes. First, the detection of very far galaxies at redshifts well beyond 6 (see e.g. Pelló et al. 2005) opens the way to detection of galaxies whose colours will be dominated by extremely metal-poor stars (Schaerer 2002, 2003). Second, as a complement to the observation of the deep Universe, the detection of nearby, very metal-poor halo stars provides very interesting clues to the early chemical evolution of our Galaxy (Beers et al. 1992; Beers 1999; Christlieb et al. 2004; Bessell et al. 2004; Cayrel et al. 2004; Spite et al. 2005). These works have shown the following very interesting results:

- The measured abundances of many elements at very low metallicity present a very small scatter (Cayrel et al. 2004). At first sight this appears difficult to understand. Indeed, in the early Universe, stars are believed to form from the ejecta of a small number of supernovae (may be only one). For instance the Argast et al. models (2000, 2002) predict that for $[\mathrm{Fe} / \mathrm{H}]<-3$, type II supernovae enrich the interstellar medium only locally. Since the chemical composition of supernova ejecta may differ a lot from case to case, large scatter of the abundances is expected at very low metallicity. For most of the elements, however, this strong scatter is not observed, at least down to a metallicity of $[\mathrm{Fe} / \mathrm{H}] \sim-4.0$ (Cayrel et al. 2004). This might indicate that, already at this low metallicity, stars are formed from a wellmixed reservoir composed of ejecta from stars of different initial masses.

- These observations also show that there is no sign of enrichments by pair-instability supernovae, at least down to a metallicity of $[\mathrm{Fe} / \mathrm{H}]$ equal to -4 . Let us recall that these supernovae have very massive stars as progenitors, with initial masses between approximately 140 and $260 M_{\odot}$ (Barkat et al. 1967; Bond et al. 1984; Heger \& Woosley 2002). Such massive stars are believed to form only in very metal-poor environments. At the end of their lifetime, they are completely destroyed when they explode as a pair-instability supernova. In this way they may strongly contribute to the enrichment of the primordial interstellar medium in heavy elements. The composition of the ejecta of pair-instability 
supernovae is characterised by a well marked odd-even effect and a strong zinc deficiency. These two features are not observed in the abundance pattern of very metal-poor halo stars. Does this mean that at $[\mathrm{Fe} / \mathrm{H}]$ equal to -4 , pair-instability supernovae no longer dominate the chemical evolution of galaxies or that such stars are not formed? If formed, could these stars skip the pair instability or have different nucleosynthetic outputs from those currently predicted by theoretical models?

- The N/O ratios observed at the surface of halo stars by Israelian et al. (2004) and Spite et al. (2005) indicate that important amounts of primary nitrogen should be produced by very metal-poor massive stars (Chiappini et al. 2005). The physical conditions for such important productions of primary nitrogen by very metal-poor massive stars remain to be found.

- If most stars at a given $[\mathrm{Fe} / \mathrm{H}]$ present a great homogeneity in composition, a small group, comprising about 20-25\% of the stars with $[\mathrm{Fe} / \mathrm{H}]$ below -2.5 , show very large enrichments in carbon. These stars are known as C-rich extremely metal-poor (CEMP) stars. The observed [C/Fe] ratios are between $\sim 2$ and 4 , showing a large scatter. Other elements, such as nitrogen and oxygen (at least in the few cases where the abundance of this element could be measured), are also highly enhanced. Interestingly, the two most metal-poor stars known up to now, the Christlieb star or HE 0107-5240, a halo giant with $[\mathrm{Fe} / \mathrm{H}]=-5.3$, and the subgiant or mainsequence star $\mathrm{HE} 1327-2326$ with $[\mathrm{Fe} / \mathrm{H}]=-5.4$ (Frebel et al. 2005) belong in this category. To explain such high and scattered CNO abundances, obviously a special process has to be invoked (see Sect. 6 below).

The results outlined above clearly indicate that new scenarios for the formation and evolution of massive stars at very low $Z$ need to be explored.

Among the physical ingredients that could open new evolutionary paths in very metal-poor environments, rotation certainly appears a very interesting possibility. First, for metallicities $Z$ between 0.004 and 0.040 , the inclusion of rotation improves the agreement between the models and observations in many respects by allowing us to reproduce the observed surface abundances (Heger \& Langer 2000; Meynet \& Maeder 2000), the ratio of blue-to-red supergiants at low metallicity (Maeder \& Meynet 2001), the variation with the metallicity of the WR/O ratios and of the numbers of type Ibc to type II supernovae (Meynet \& Maeder 2003, 2005). Most likely, stars are also rotating at very low metallicity, and one can hope that the same physical model assumptions that improve the physical description of stars at $Z \geq 0.004$ would also apply to the very low metallicity domain.

Second, if the effects of rotation are already quite significant at high metallicity, one expects that they are even more important at lower metallicity. For instance, it was shown in previous works that the chemical mixing becomes more efficient for lower metallicity for a given initial mass and velocity (Maeder \& Meynet 2001; Meynet \& Maeder 2002). This comes from the fact that the gradients of $\Omega$ are much steeper in the lower metallicity models, so they trigger more efficient shear mixing. The gradients are steeper because less angular momentum is transported outwards by the meridional currents, whose velocity scales as the inverse of the density in the outer layers (see the Gratton-Öpick term in the expression for the meridional velocity in Maeder \& Zahn 1998).

Third, rotation can induce mass loss in two ways. The first way, paradoxically, is linked to the fact that very metal-poor stars are believed to lose little mass by radiatively driven stellar winds. Indeed, in the radiatively driven wind theory, the mass loss scales with the metallicity of the outer stellar layers as $\left(Z / Z_{\odot}\right)^{\alpha}$ with $\alpha$ between 0.5 and 0.8 (Kudritzki et al. 1987; Vink et al. 2001). Thus lowering the metallicity by a factor 200000 (as would be the case for obtaining the metallicity of the Christlieb star) would thus lower the mass loss rates by a factor 450 , or even by a greater factor if the metallicity dependence becomes stronger at lower $Z$, as suggested by Kudritzki (2002). Now since metal-poor stars lose little mass, they also lose little angular momentum (if rotating), so they have a greater chance of reaching the break-up limit during the Main Sequence phase (see for instance Fig. 9 in Meynet \& Maeder 2002). At break-up, the outer stellar layers become unbound and are ejected whatever their metallicity. The break-up is reached more easily when we take into account that massive rotating stars have polar winds as shown by Owocki et al. (1996) and Maeder (1999). When most of the mass is lost along the rotational axis, little angular momentum is lost.

Another way for rotation to trigger enhancements of the mass loss comes from the mixing induced by rotation. In general, rotational mixing favours the evolution into the red supergiant stage (see Maeder \& Meynet 2001), where mass loss is higher. It also enhances the metallicity of the surface of the star and, in this way, boosts the radiatively driven stellar winds (see below).

Thus there are very good reasons for exploring the effects of rotation at very low metallicity, which we have attempted in this work. This was also the aim of the recent work by Marigo et al. (2003), who compute Pop III massive stellar models with rotation, assuming solid-body rotation. In this very interesting piece of work, they mainly study the effects of reaching the break-up limit. However, since they did not include the rotational mixing of the chemical elements, they could not explore the effects of rotation on the internal chemical composition of the stars. Also, solid body rotation is just the extreme case of coupling the internal rotation, which ignores the physics and timescales of the internal transport. In the present models, the transport of both the angular momentum and the chemical species are treated in a consistent way, and, as we shall see, rotational mixing has very important consequences on both the stellar yields and the mass loss rates.

In Sect. 2, we briefly recall the main physical ingredients of the models. The evolutions of fast-rotating massive star models at very low $Z$ are described in Sect. 3. The evolution of the internal chemical composition is the subject of Sect. 4, while the ejected masses of various isotopes are presented in Sect. 5. The case of the CEMP stars is discussed in Sect. 6. Section 7 summarises the main results and raises a few questions to be explored in future works. 


\section{Physical ingredients}

The computation of our models was done with the Geneva evolution code. The opacities were taken from Iglesias \& Roger (1996) and complemented at low temperatures by the molecular opacities of Alexander (http://web.physics.twsu.edu/alex/wwwdra.htm). The nuclear reaction rates were based on the NACRE data basis (Angulo et al. 1999). The treatment of rotation included the hydrostatic effects described in Meynet \& Maeder (1997) and the effects of rotation on mass loss rates according to Maeder \& Meynet (2000). In particular, we accounted for the wind anisotropies induced by rotation as in Maeder (1999). Meridional circulation was implemented according to Maeder \& Zahn (1998), but including the new $D_{\mathrm{h}}$ coefficient as described in Maeder (2003). Roughly compared to the old $D_{\mathrm{h}}$, the new one tends to reduce the size of the convective core and to allow larger enrichment of the surface in CNO-processed elements. The reader is referred to these papers for a detailed description of the effects. The convective instability was treated according to the Schwarzschild criterion without overshooting. The radiative mass loss rates are from Kudritzki $\&$ Puls (2000) when $\log T_{\text {eff }}>3.95$ and from de Jager et al. (1988) otherwise. The mass loss rates depend on metallicity as $\dot{M} \sim\left(Z / Z_{\odot}\right)^{0.5}$, where $Z$ is the mass fraction of heavy elements at the surface of the star. As we shall see, this quantity may change during the evolution of the star.

A specific treatment for mass loss was applied at break-up. At break-up, the mass loss rate adjusts itself in such a way that an equilibrium is reached between the two following opposite effects. 1) The radial inflation due to evolution, combined with the growth of the surface velocity due to the internal coupling by meridional circulation, brings the star to break-up, and thus some amount of mass at the surface is no longer bound to the star. 2) By removing the most external layers, mass loss brings the stellar surface down to a level in the star that is no longer critical. Thus, at break-up, we should adapt the mass loss rates, in order to maintain the surface layers at the break-up limit. In practice, however, since the critical limit contains mathematical singularities, we considered that during the break-up phase, the mass loss rates should be such that the model stays near a constant fraction (for example, 0.98) of the limit. At the end of the MS phase, the stellar radius inflates so rapidly that meridional circulation is unable to continue to ensure the internal coupling, and the break-up phase ceases naturally.

In this first exploratory work, we focused our attention on stars with initial masses of $60 M_{\odot}$ and $7 M_{\odot}$ in order to gain insight into the properties of both massive and AGB stars at low $Z$. The evolution was computed until the end of the core helium burning phase (core carbon burning phase in the case of the $60 M_{\odot}$ rotating model at $Z=10^{-8}$ ). Two metallicities were considered for the $60 M_{\odot}$ models: $Z=10^{-8}$ and $Z=$ $10^{-5}$. Only this last metallicity was considered for the $7 M_{\odot}$ model. Of course we do not know if stars with $Z=10^{-8}$ have ever formed; however, it is not possible at the present time to exclude such a possibility. Indeed it might be that the first star generations produce very little amounts of heavy elements, due to the strong fallback of ejected material onto black holes at the
Table 1. Initial abundances in mass fraction for models at $Z=10^{-8}$.

\begin{tabular}{cc}
\hline \hline Element & Initial abundance \\
\hline $\mathrm{H}$ & 0.75999996 \\
${ }^{3} \mathrm{He}$ & 0.00002554 \\
${ }^{4} \mathrm{He}$ & 0.23997448 \\
${ }^{12} \mathrm{C}$ & $7.5500 \mathrm{e}-10$ \\
${ }^{13} \mathrm{C}$ & $0.1000 \mathrm{e}-10$ \\
${ }^{14} \mathrm{~N}$ & $2.3358 \mathrm{e}-10$ \\
${ }^{15} \mathrm{~N}$ & $0.0092 \mathrm{e}-10$ \\
${ }^{16} \mathrm{O}$ & $67.100 \mathrm{e}-10$ \\
${ }^{17} \mathrm{O}$ & $0.0300 \mathrm{e}-10$ \\
${ }^{18} \mathrm{O}$ & $0.1500 \mathrm{e}-10$ \\
${ }^{19} \mathrm{~F}$ & $0.0020 \mathrm{e}-10$ \\
${ }^{20} \mathrm{Ne}$ & $7.8368 \mathrm{e}-10$ \\
${ }^{21} \mathrm{Ne}$ & $0.0200 \mathrm{e}-10$ \\
${ }^{22} \mathrm{Ne}$ & $0.6306 \mathrm{e}-10$ \\
${ }^{23} \mathrm{Na}$ & $0.0882 \mathrm{e}-10$ \\
${ }^{24} \mathrm{Mg}$ & $3.2474 \mathrm{e}-10$ \\
${ }^{25} \mathrm{Mg}$ & $0.4268 \mathrm{e}-10$ \\
${ }^{26} \mathrm{Mg}$ & $0.4897 \mathrm{e}-10$ \\
${ }^{27} \mathrm{Al}$ & $0.1400 \mathrm{e}-10$ \\
${ }^{28} \mathrm{Si}$ & $3.2800 \mathrm{e}-10$ \\
${ }^{56} \mathrm{Fe}$ & $3.1675 \mathrm{e}-10$ \\
\hline
\end{tabular}

end of their lifetimes. Moreover, as we shall see, the behaviour of the $Z=10^{-5}$ and $10^{-8}$ massive star models are qualitatively similar, indicating that the evolutionary scenarios explored here might apply to a broad range of initial metallicities.

The initial mixture of heavy elements was taken as equal to the one used to compute the opacity tables (Iglesias \& Roger 1996, Weiss alpha-enhanced elements mixture). The initial composition for models at $Z=10^{-8}$ is given in Table 1 . The models at $Z=10^{-5}$ have the same initial mixture of heavy elements. More precisely, the mass fractions for all the isotopes heavier than ${ }^{4} \mathrm{He}$ were multiplied by $10^{3}\left(=10^{-5} / 10^{-8}\right)$.

Nothing is known on the rotational velocities of such stars. However, there are some indirect indications that stars at lower $Z$ could have higher initial rotational velocities: 1 ) realistic simulations of the formation of the first stars in the Universe show that the problem of the dissipation of the angular momentum is more severe at very low $Z$ than at the solar $Z$. Thus these stars might begin their evolution with a higher amount of angular momentum (Abel et al. 2002). 2) There are some observational hints that the distribution of initial rotation might contain more fast rotators at lower $Z$ (Maeder et al. 1999). 3) Even if stars begin their life on the ZAMS with the same total amount of angular momentum for all metallicities, then the stars at lower metallicity rotate faster as a consequence of their smaller radii.

The three arguments listed above would favour the choice of a higher value for the initial rotational velocity than those adopted for solar models. To choose this value we proceeded in the following way. First we supposed that the stars begin their evolution on the ZAMS with approximately the same 
Table 2. Properties of the stellar models at the end of the $\mathrm{H}$ - and He-burning phases. $M_{\text {ini }}$ is the initial mass, $Z$ the initial metallicity, $v_{\text {ini }}$ the initial velocity, $\bar{v}$ the mean equatorial rotational velocity during the MS phase defined as in Meynet \& Maeder (2000), $t_{\mathrm{H}}$ the H-burning lifetimes, $M$ the actual mass of the star, $v$ the actual rotational velocity at the stage considered, $Y_{\mathrm{s}}$ the helium surface abundance in mass fraction. N/C and N/O are the ratios of nitrogen to carbon, respectively, of nitrogen to oxygen at the surface of stars in mass fraction; C, N, O are the abundances of carbon, nitrogen, and oxygen at the surface in mass fractions. The numbers in parentheses indicate the power of ten, i.e., $7.54(-10)=7.54 \times 10^{-10}$.

\begin{tabular}{|c|c|c|c|c|c|c|c|c|c|c|c|c|c|c|c|c|}
\hline \multirow[b]{2}{*}{$\begin{array}{c}M_{\text {ini }} \\
M_{\odot}\end{array}$} & \multirow[b]{2}{*}{$Z$} & \multirow[b]{2}{*}{$\begin{array}{c}v_{\text {ini }} \\
\mathrm{km} \mathrm{s}^{-1} \\
\end{array}$} & \multirow[b]{2}{*}{$\begin{array}{c}\bar{v} \\
\mathrm{~km} \mathrm{~s}^{-1} \\
\end{array}$} & \multicolumn{6}{|c|}{ End of H-burning } & \multicolumn{7}{|c|}{ End of He-burning } \\
\hline & & & & $\begin{array}{c}t_{\mathrm{H}} \\
\mathrm{Myr}\end{array}$ & $\begin{array}{c}M \\
M_{\odot} \\
\end{array}$ & $\begin{array}{c}v \\
\mathrm{~km} \mathrm{~s}^{-1}\end{array}$ & $Y_{\mathrm{s}}$ & $\mathrm{N} / \mathrm{C}$ & $\mathrm{N} / \mathrm{O}$ & $\begin{array}{c}t_{\mathrm{He}} \\
\mathrm{Myr}\end{array}$ & $\begin{array}{c}M \\
M_{\odot} \\
\end{array}$ & $\begin{array}{c}v \\
\mathrm{~km} \mathrm{~s}^{-1}\end{array}$ & $Y_{\mathrm{s}}$ & $\mathrm{C}$ & $\mathrm{N}$ & $\mathrm{O}$ \\
\hline 60 & $10^{-8}$ & 0 & 0 & 3.605 & 59.817 & 0 & 0.24 & 0.31 & 0.03 & 0.292 & 59.726 & 0 & 0.24 & $7.54(-10)$ & $2.34(-10)$ & $6.71(-9)$ \\
\hline 60 & $10^{-8}$ & 800 & 719 & 4.004 & 57.624 & 591 & 0.27 & 103 & 9.77 & 0.522 & 23.988 & 0.02 & 0.76 & $1.97(-4)$ & $1.02(-2)$ & $2.85(-4)$ \\
\hline 60 & $10^{-5}$ & 800 & 636 & 4.441 & 53.846 & 567 & 0.34 & 40 & 0.82 & 0.544 & 37.280 & 0.57 & 0.80 & $5.06(-5)$ & $2.07(-3)$ & $4.24(-5)$ \\
\hline
\end{tabular}

angular momentum content, whatever the metallicity. At solar metallicity, observation provides values for the mean observed rotational velocity on the MS phase (around $200 \mathrm{~km} \mathrm{~s}^{-1}$ for OB stars). Stellar models allowed us to estimate the initial angular momentum required to achieve such values (around $2.2-2.5 \times 10^{53} \mathrm{~g} \mathrm{~cm}^{2} \mathrm{~s}^{-1}$ ). Adopting such an initial value of the angular momentum, we found that a $60 M_{\odot}$ stellar model at $Z=10^{-8}$ has a velocity on the ZAMS of $800 \mathrm{~km} \mathrm{~s}^{-1}$. This is the value of the initial velocity we adopt in the present work.

\section{Evolution of a massive rotating star at very low metallicity}

\subsection{Rotation and mass loss during the main sequence phase}

Figure 1 shows the evolutionary tracks during the main sequence (MS) phase for the $60 M_{\odot}$ stellar models at $Z=10^{-8}$. Table 2 presents some properties of the models at the end of the core $\mathrm{H}$ - and He-burning phases. From Fig. 1, we see that rotation produces a small shift of the tracks toward lower luminosities and $T_{\text {eff }}$. This effect is due to both atmospheric distortions (note that surface-averaged effective temperatures are plotted in Fig. 1 as explained in Meynet \& Maeder 2000) and to the lowering of the effective gravity (see e.g. Kippenhahn \& Thomas 1970; Maeder \& Peytremann 1970; Collins \& Sonneborn 1977). The MS lifetime of the rotating model is enhanced by $11 \%$.

These results show that rotation does not affect the UV outputs of very metal-poor massive stars much (the UV outputs of the first massive star generations might contribute a lot to the reionization of the early Universe, see e.g. Madau 2003). Only if a significant fraction of primordial stars would rotate so fast that they follow the path of homogeneous evolution (Maeder 1987), could rotation increase the ionizing power. In that case, the star would remain in the blue part of the HR diagram and would have a much longer lifetime.

Figure 2 shows the evolution of the ratio $\Omega / \Omega_{\text {crit }}$ at the surface during the MS phase. At $Z=10^{-8}$, the model with $v_{\text {ini }}=800 \mathrm{~km} \mathrm{~s}^{-1}$ reaches the break-up limit when the mass fraction of hydrogen at the centre $X_{\mathrm{c}} \simeq 0.40$. The star stays at break-up for the remaining of its MS life with an enhanced

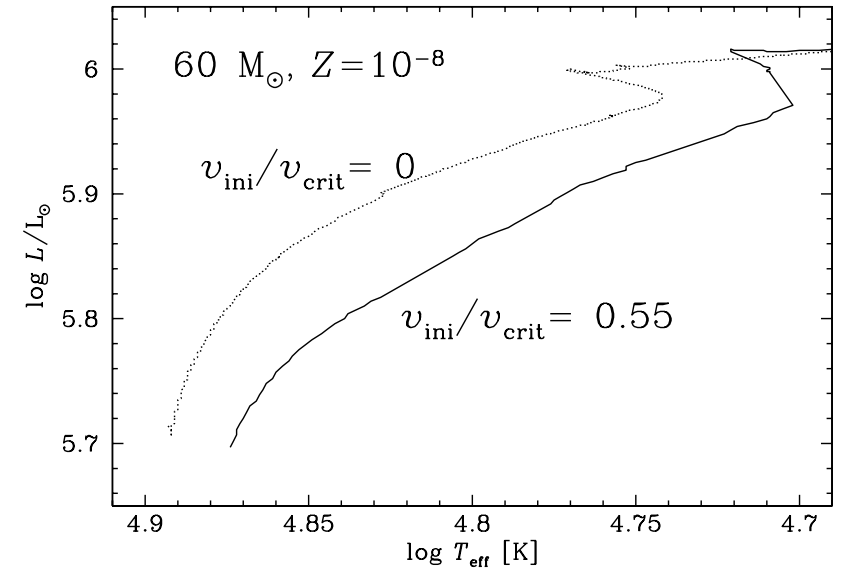

Fig. 1. Evolutionary tracks in the HR diagram for $60 M_{\odot}$ stellar models at $Z=10^{-8}$.

mass loss rate. As a consequence, the model ends its MS life with 57.6 $M_{\odot}$, having lost $4 \%$ of its initial mass. Despite the star staying in the vicinity of the break-up limit during an important part of its MS lifetime, it does not lose very large amounts of mass, due to the fact that only the outermost layers of the stars are above the break-up limit and are ejected. These layers have low density and thus contain little mass.

A model with the same initial velocity, but with a metallicity three orders of magnitude higher, reaches the break-up limit very early in the MS phase, when $X_{\mathrm{c}} \simeq 0.56$. This comes from the fact that when the metallicity increases, a given value of the initial velocity corresponds to a higher initial value of the $v_{\text {ini }} / v_{\text {crit }}$ ratio. The model at $Z=10^{-5}$ ends its MS life with $53.8 M_{\odot}$, having lost $10 \%$ of its initial mass.

In order to discuss the effects of a change of rotation, it is interesting to compare this last result with the one obtained in Meynet \& Maeder (2002) for a $60 M_{\odot}$ model at $Z=10^{-5}$ with $v_{\text {ini }}=300 \mathrm{~km} \mathrm{~s}^{-1}$. This last model reaches the break-up velocity much later, only when $X_{\mathrm{c}} \simeq 0.01$. At $Z=10^{-5}$, the velocity $300 \mathrm{~km} \mathrm{~s}^{-1}$ thus appears as the lower limit for the initial rotation, allowing a $60 M_{\odot}$ star to reach the break-up limit during its MS phase. The $60 M_{\odot}$ star ends its MS life with $59.7 M_{\odot}$, having lost only $0.5 \%$ of its initial mass. Note that the 2002 models were computed with slightly different 


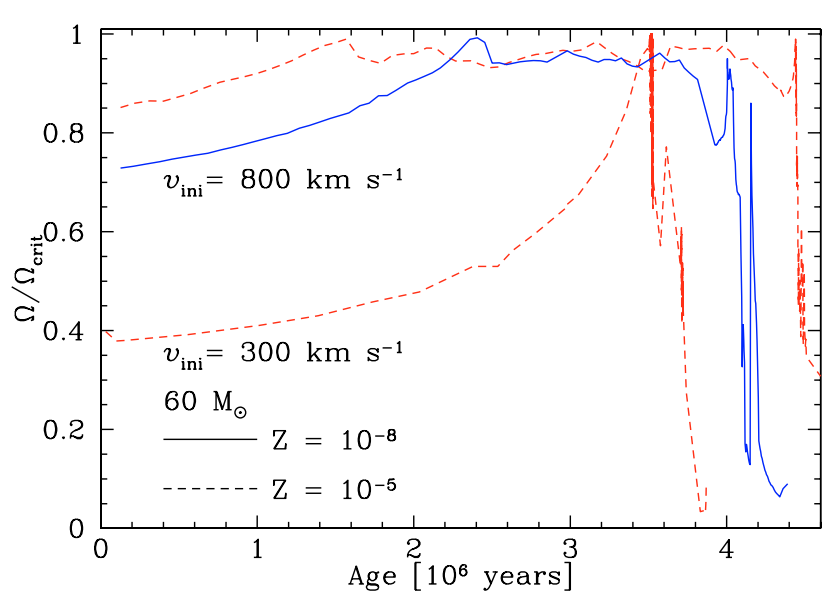

Fig. 2. Evolution of $\Omega / \Omega_{\text {crit }}$ at the surface of $60 M_{\odot}$ models at $Z=10^{-8}$ (continuous line) and $Z=10^{-5}$ (upper dashed line) with $v_{\text {ini }}=$ $800 \mathrm{~km} \mathrm{~s}^{-1}$. The case of the $60 M_{\odot}$ model at $Z=10^{-5}$ with $v_{\text {ini }}=$ $300 \mathrm{~km} \mathrm{~s}^{-1}$ from Meynet \& Maeder (2002) is also shown (lower dashed line).

physical ingredients than those used to compute the models discussed in this paper (different expression for $D_{\mathrm{h}}$ and for the mass loss rates). However, at these very low metallicities, radiatively driven winds remain quite modest, and the transport of the angular momentum, mainly driven by the meridional circulation, does not depend much on the expression of $D_{\mathrm{h}}$.

During the MS phase, the surface of the rotating stars is enriched in nitrogen and depleted in carbon as a result of rotational mixing. Figure 3 shows that the N/C ratios are enhanced by more than two orders of magnitude at the end of the H-burning phase. More precisely, at the surface of the $Z=10^{-8}$ model, nitrogen is enhanced by a factor 27 and carbon decreased by a factor 12 . All other physical ingredients being the same, one also sees that the model with the lowest metallicity is also the one with the greatest surface enrichments. This well agrees with the trend already found in our previous works (Maeder \& Meynet 2001; Meynet \& Maeder 2002), which results from the steep gradients of angular velocity that build up in very metal-poor stars that favours shear mixing. Let us emphasise, however, that during the MS phase, the arrival of CNO-processed material at the surface does not change the metallicity of the outer layers. Indeed, rotational mixing brings nitrogen to the surface but depletes carbon and oxygen, keeping the sum of CNO elements constant, and the metallicity as well. Thus during the MS phase, the enhanced mass loss rates undergone by the rotating models are entirely due to the mechanical effect of the centrifugal force. As we shall see, this is no longer the case during the core He-burning phase.

\subsection{Rotation and mass loss during the post main-sequence phases}

Rotational mixing that occurs during the MS phase and still continues to be active during the core He-burning phase deeply modifies the internal chemical composition of the star. This has important consequences during the post-MS phases and deeply changes the evolution of the rotating models with respect to

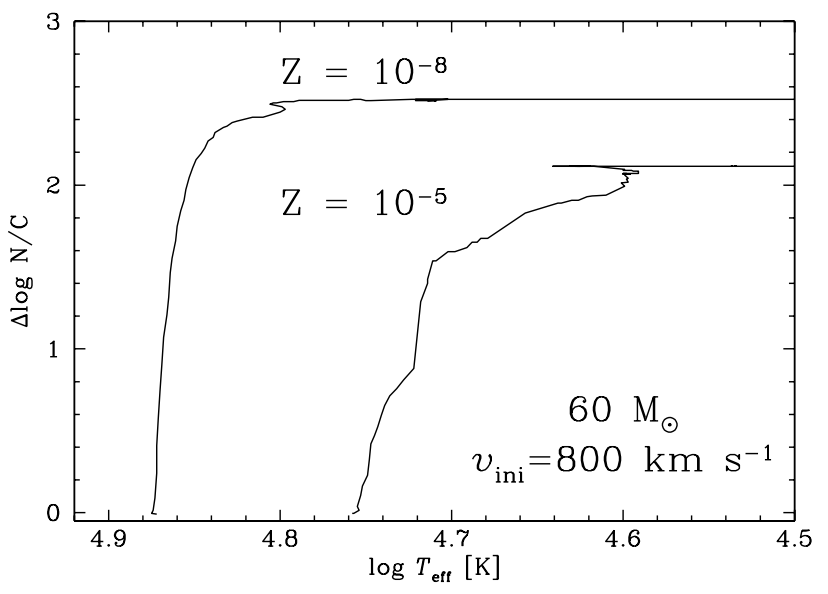

Fig. 3. Evolution as a function of $\log T_{\text {eff }}$ of the excess at the surface for the ratio $\mathrm{N} / \mathrm{C}$ (expressed in dex) compared to the initial ratio. $\mathrm{N}$ and $\mathrm{C}$ are the abundances of nitrogen and carbon at the surface of the star.

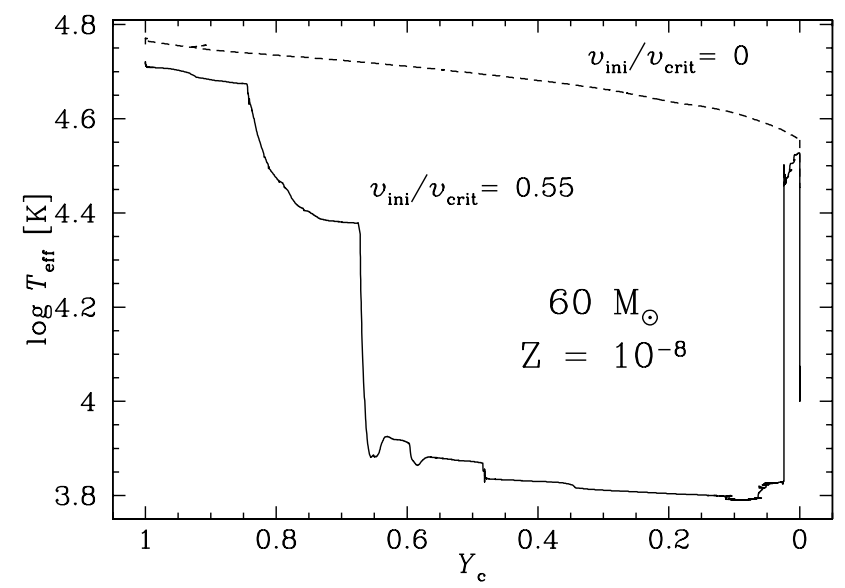

Fig. 4. Evolution of $\log T_{\text {eff }}$ as a function of $Y_{\mathrm{c}}$, the mass fraction of ${ }^{4} \mathrm{He}$ at the centre, for a non-rotating (dashed line) and rotating (continuous line) $60 M_{\odot}$ model at $Z=10^{-8}$.

their non-rotating counterparts. Among the most striking differences, one notes the following:

1) Rotation favours redwards evolution in the HR diagram as was already shown by Maeder \& Meynet (2001), and as illustrated in Fig. 4. One sees that the non-rotating model remains on the blue side during the whole core He-burning phase, while the $800 \mathrm{~km} \mathrm{~s}^{-1}$ model at $Z=10^{-8}$ starts its journey toward the red side of the HR diagram early in the core helium burning stage, when $Y_{\mathrm{c}} \simeq 0.67$ ( $Y_{\mathrm{c}}$ is the mass fraction of helium at the centre of the star model). The same is true for the corresponding model at $Z=10^{-5}$. Let us recall that this behaviour is linked to the rapid disappearance of the intermediate convective zone associated to the H-burning shell (see Fig. 5 and Maeder \& Meynet 2001).

2) Redwards evolution enhances the mass loss. In the cases of our $60 M_{\odot}$ stellar models, it brings the stars near the Humphreys-Davidson limit, i.e., near $\log L / L_{\odot}=6$ and $T_{\text {eff }}$ in a broad range around $10^{4} \mathrm{~K}$. Near this limit, the mass loss rates (here from de Jager et al. 1988) become very important. For instance, the model represented in the left 


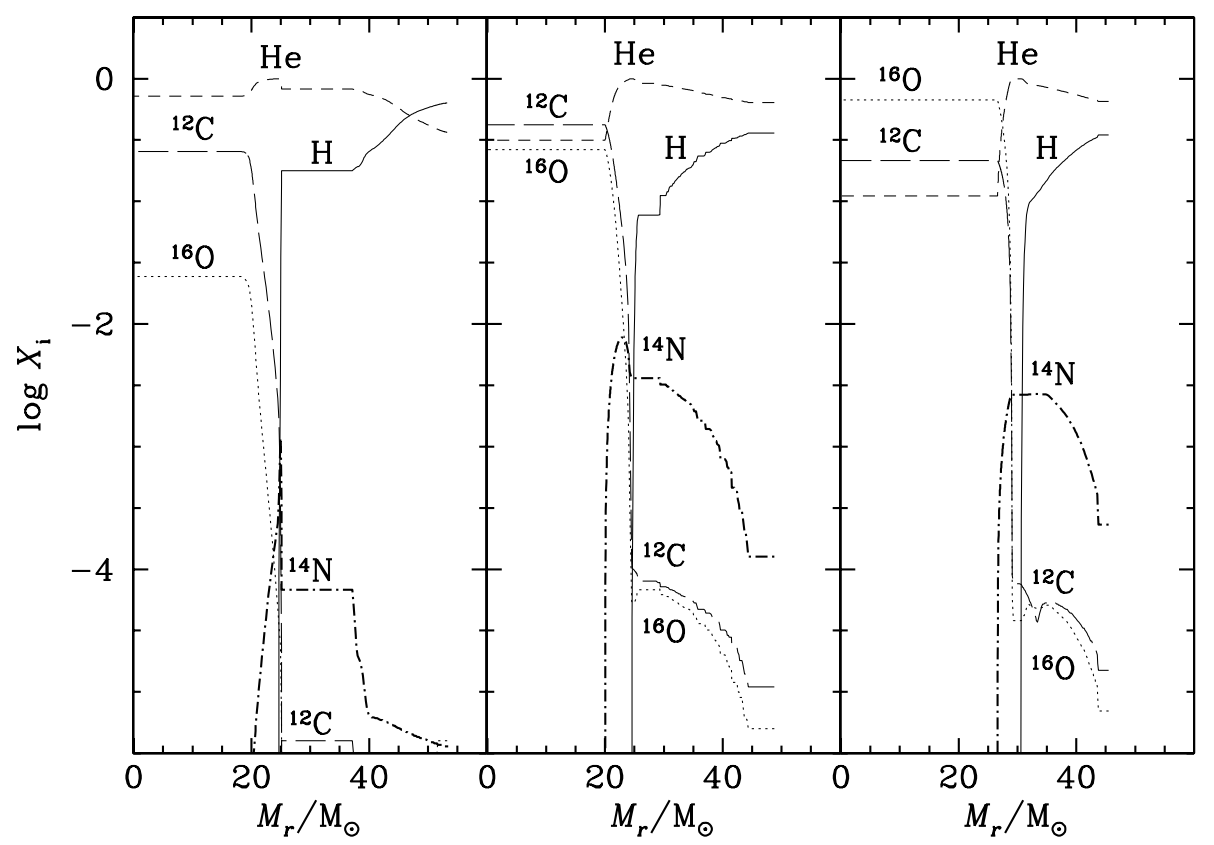

Fig. 5. Chemical composition of a $60 M_{\odot}$ stellar model at $Z=10^{-5}$ with $v_{\text {ini }}=800 \mathrm{~km} \mathrm{~s}^{-1}$ when it evolves from the blue to the red part of the HR diagram. The model shown in the left panel has $\log L / L_{\odot}=6.129$ and $\log T_{\text {eff }}=4.243$; in the middle panel, it has $\log L / L_{\odot}=6.130$ and $\log T_{\text {eff }}=4.047$; in the right panel, it has $\log L / L_{\odot}=6.145$ and $\log T_{\text {eff }}=3.853$.

panel of Fig. $5\left(\log L / L_{\odot}=6.129, \log T_{\text {eff }}=4.243\right)$ is still far to the left hand side of the Humphreys-Davidson limit. Its mass loss rate is $\log (-\dot{M})=-5.467$, where $\dot{M}$ is expressed in $M_{\odot}$ per year. The model in the right panel $\left(\log L / L_{\odot}=6.145, \log T_{\text {eff }}=3.853\right)$ is in the vicinity of the Humphreys-Davidson limit. Its mass loss rate is equal to -4.616 , i.e., more than seven times higher. During this transition, the overall metallicity at the surface does not change and remains equal to the initial one (here $Z_{\text {ini }}=$ $0.00001)$. We observe a similar transition in the case of the $Z=10^{-8}$ stellar model.

3) During the core He-burning phase, primary nitrogen is synthesized in the H-burning shell, due to the rotational diffusion of carbon and oxygen produced in the helium core into the H-burning shell (Meynet \& Maeder 2002). This is illustrated well in Fig. 5 for the $Z=10^{-5}$ rotating model and in Fig. 6 for the model at $Z=10^{-8}$.

4) In contrast to what happens during the MS phase, rotational mixing during the core He-burning phase induces large changes in the surface metallicity. These changes occur only at the end of the core He-burning phase, although the conditions for their apparition result from the mixing that occurs during the whole core He-burning phase. Indeed, rotational mixing progressively enriches the outer radiative zone in CNO elements, thus enhancing its opacity slowly. When, in the $Z=10^{-8}$ stellar model, the abundance of nitrogen in the outer layers becomes approximately $10^{-8}$ in mass fraction (i.e., has increased by two orders of magnitude with respect to the initial value), these outer layers become convective. The outer convective zone then rapidly deepens in mass and dredges up newly synthesized elements to the surface. From this stage onwards, the surface metallicity increases in a spectacular way, as can be seen in Fig. 6. For instance, the rotating $60 M_{\odot}$ at $Z=10^{-8}$ has a surface metallicity of $10^{-2}$ at the end of its lifetime, i.e., similar to that of the Large Magellanic Cloud!

5) The consequence of such large surface enrichments on the mass loss rates remains to be studied in detail using models of stellar winds with the appropriate physical characteristics (position in the HR diagram and chemical composition). In the absence of such sophisticated models, we applied the usual rule here, namely $\dot{M}(Z)=\left(Z / Z_{\odot}\right)^{1 / 2} \dot{M}\left(Z_{\odot}\right)$, where $Z$ is the metallicity of the outer layers. With this prescription, the surface enhancement of the metallicity is responsible for the large decrease in the stellar mass that can be seen in Fig. 6.

6) During the late stages of the core helium-burning phase, as a result of mass loss and mixing, the star may evolve along a blue loop in the HR diagram (see Fig. 4). When the star evolves bluewards, the global stellar contraction brings the outer convective zone, which evolves like a solid body rotating shell to break-up (Heger \& Langer 1998). At this stage of the evolution, the luminosity is not far from the Eddington limit and the star may reach the $\Omega \Gamma$-limit (Maeder \& Meynet 2000). This multiplies the mass loss rates by very large factors.

7) During the last 24000 years of its lifetime, the model presents abundance patterns characteristic of WNL stars at its surface.

8) As a result of mixing and mass loss rates, the duration of the core He-burning phase is much longer in the rotating model. The present $60 M_{\odot}$ model with $v_{\text {ini }}=800 \mathrm{~km} \mathrm{~s}^{-1}$ at $Z=10^{-8}$ has a helium-burning lifetime that is $\sim 80 \%$ longer than the corresponding lifetime of the non-rotating model (see Sect. 4 below for more explanations). 


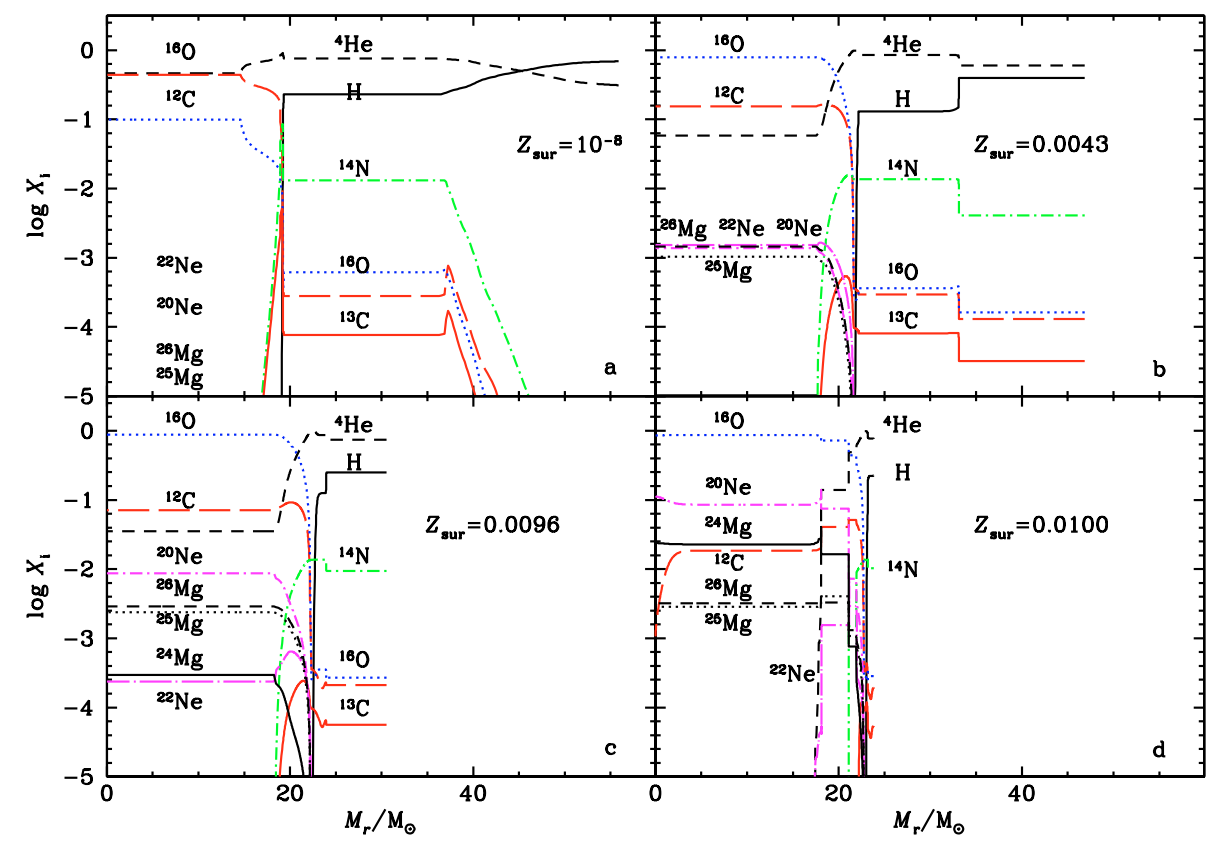

Fig. 6. Variations of the abundances (in mass fraction) as a function of the Lagrangian mass within a $60 M_{\odot}$ star with $v_{\text {ini }}=800 \mathrm{~km} \mathrm{~s}^{-1}$ and $Z=10^{-8}$. The four panels show the chemical composition at four different stages at the end of the core He-burning phase: in panel a) the model has a mass fraction of helium at the centre, $Y_{\mathrm{c}}=0.11$ and an actual mass $M=54.8 M_{\odot}$. b) $Y_{\mathrm{c}}=0.06, M=48.3 M_{\odot}$. c) $Y_{\mathrm{c}}=0.04, M=31.5 M_{\odot}$. d) End of the core C-burning phase, $M=23.8 M_{\odot}$. The actual surface metallicity $Z_{\text {surf }}$ is indicated in each panel.

The different effects described above are all due to the mixing induced by rotation and they all tend to enhance the quantity of mass lost by stellar winds. The rotating $60 M_{\odot}$ at $Z=10^{-8}$ loses about $36 M_{\odot}$ during its lifetime. About $2 M_{\odot}$ are lost due to break-up during the MS phase, $\sim 3 M_{\odot}$ are lost when the star is in the red part of the HR diagram (with surface metallicity equal to the initial one), $27 M_{\odot}$ are lost due to the effect of the enhancement of the surface metallicity, the remaining $4 M_{\odot}$ are lost when the star evolves along the blue loop and reaches the $\Omega \Gamma$-limit. One sees that, by far, the most important effect is due to the increase in the surface metallicity.

Paradoxically the corresponding model at higher metallicity $\left(Z=10^{-5}\right)$ loses less mass (a little less than $40 \%$ of the total mass). This can be understood from the following facts: first less primary nitrogen is synthesized due to slightly less efficient chemical mixing when the metallicity increases, thus the surface does not become as metal rich as in the model at $Z=10^{-8}$. Second and for the same reason as above, the outer convective zone does not deepen as far as in the more metal-poor model. These two factors imply that the maximum surface metallicity reached in this model, which is equal to 0.0025 , is about a factor 4 below the one reached by the $Z=10^{-8}$ model. Finally, the blue loop does not extend that far into the blue side, and the surface velocity always remains well below the break-up limit during the whole blueward excursion.

In order to investigate to what extent the behaviour described above depends on the physical ingredients of the model, we compare the present results with those of a rotating model $\left(v_{\text {ini }}=800 \mathrm{~km} \mathrm{~s}^{-1}\right)$ of a $60 M_{\odot}$ star at $Z=10^{-5}$ with a different prescription for the mass loss rates (Vink et al. 2000, 2001 instead of Kudritzki \& Puls 2000), with the Ledoux criterion instead of the Schwarzschild one for determining the size of the convective core, with a core overshoot of $\alpha=0.2 H_{p}$ and the old prescription for the horizontal diffusion coefficient $D_{\mathrm{h}}$. This model is described in Meynet et al. (2005). In this case, the outer convective zone deepens farther into the stellar interior and thus produces a greater enhancement of the surface metallicity (the same order as the one we obtained in the present $Z=10^{-8} 60 M_{\odot}$ model). Higher enhancements of the surface metallicity then induces greater mass loss by stellar winds. More important than these differences, however, we shall retain here that the results are qualitatively similar to those obtained in our previous models. In particular, the mechanism of surface metallicity enhancement occurs in both models and appears to be a robust process.

\subsection{Do very metal-poor, very massive stars end their lives as pair-instability supernovae?}

Might the important mass loss undergone by the rotating models prevent the most massive stars from going through pair instability? According to Heger \& Woosley (2002), progenitors of pair-instability supernovae have helium core masses between $\sim 64$ and $133 M_{\odot}$. This corresponds to initial masses between about 140 and $260 M_{\odot}$. Thus the question is whether stars with initial masses above $140 M_{\odot}$ can lose a sufficient amount of mass to have a helium core that is less than about $64 M_{\odot}$ at the end of the core He-burning phase. From the values quoted above, it would imply the loss of more than (140-64) = $76 M_{\odot}$, which represents about $54 \%$ of the initial stellar mass. From the computation performed here, one can expect that such a scenario is possible, where a $60 M_{\odot}$ loses more than $60 \%$ of its initial mass. However, more extensive computations are needed to check whether the rotational mass loss could indeed 
prevent the most massive stars from going through this pair instability. Were this the case, it would explain why the nucleosynthetic signature of pair-instability supernovae is not observed in the abundance pattern of the most metal-poor halo stars observed up to now. At least this mechanism could restrain the mass range for the progenitors of pair-instability supernovae, pushing the minimum initial mass needed for such a scenario to occur to higher values. Let us also note that the luminosity of the star comes nearer to the Eddington limit when the initial mass increases. When rotating, such stars will then encounter the $\Omega \Gamma$-limit (Maeder \& Meynet 2000) and very likely undergo strong mass losses.

\section{Evolution of the interior chemical composition}

As discussed above, rotational mixing changes the chemical composition of stellar interiors in an important way. This is illustrated well by Figs. 6 and 7, which show the internal chemical composition of our rotating and non-rotating $60 M_{\odot}$ stellar models at four different stages at the end of the core He-burning phase (models at $Z=10^{-8}$ ).

Comparing panels a of Figs. 6 and 7, one sees that a large convective shell is associated to the H-burning shell in the rotating model, while such a shell is absent in the non-rotating model. This contrasts with what happens at the beginning of the core He-burning phase, where the intermediate convective zone associated to the H-burning shell was absent in the rotating model (or at least much smaller), while in the non-rotating model, the intermediate convective zone was well-developed (see above). Why is there this difference between the beginning and the end of the core He-burning phase? At the beginning of the core He-burning phase, the disappearance of the intermediate convective shell was a consequence of the rotational mixing that operated during the core $\mathrm{H}$-burning phase and that brought some freshly synthesized helium into this region. More helium in this region means less hydrogen and also some decrease in the opacity, both of which inhibit the development of convection (cf. Maeder \& Meynet 2001). Now, at the end of the core He-burning phase, we have He-burning products that are brought into the H-burning shell. These products, mainly carbon and oxygen, act as catalysts for the CNO cycle and make the H-burning shell more active, thus favouring convection. This mechanism enriches this zone not only in primary ${ }^{14} \mathrm{~N}$, but also in primary ${ }^{13} \mathrm{C}$. Looking at the H-rich envelope in the non-rotating model at the same stage, one sees that all these elements have much lower abundances. Actually they fall well below the minimum ordinate of the figure.

If one now compares the chemical composition of the $\mathrm{CO}$ cores when $Y_{\mathrm{c}} \sim 0.11$ (see panels a of Figs. 6 and 7), one notes the following points. First, the abundances in ${ }^{12} \mathrm{C}$, ${ }^{16} \mathrm{O}$ and ${ }^{20} \mathrm{Ne}$ are approximately equal in both the rotating and non-rotating models. This comes from the fact that the $\mathrm{CO}$ core masses are approximately the same in both models. On the other hand in the rotating model, the abundance of ${ }^{22} \mathrm{Ne}$ is greatly enhanced, as the abundances of ${ }^{25} \mathrm{Mg}$ and ${ }^{26} \mathrm{Mg}$. The abundance of ${ }^{22} \mathrm{Ne}$ results from the conversion of primary ${ }^{14} \mathrm{~N}$, which has diffused into the He-burning core. Thus the resulting high abundance of ${ }^{22} \mathrm{Ne}$ is also of primary origin. The isotopes

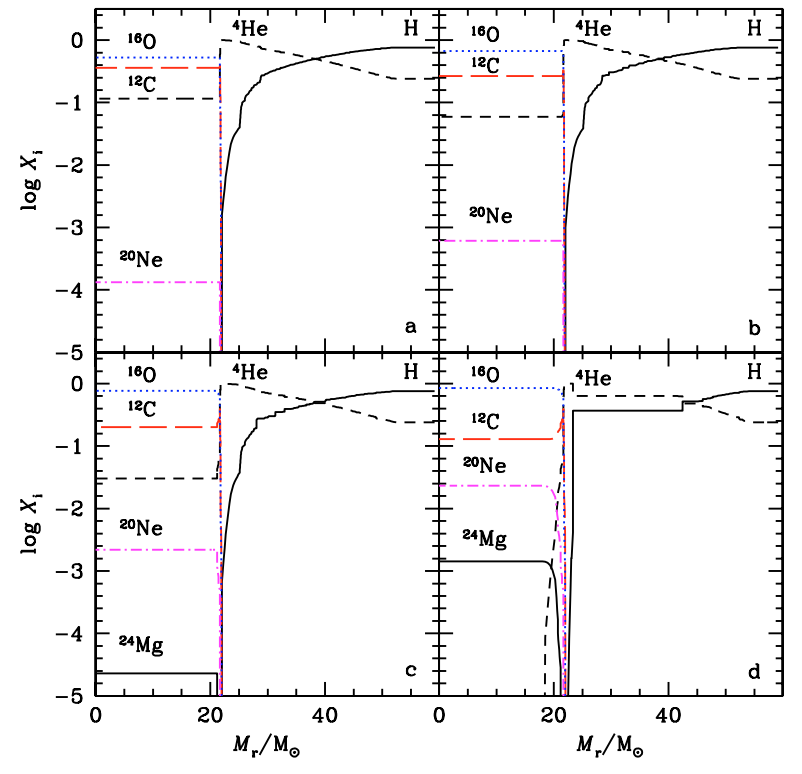

Fig. 7. Same as Fig. 6 for a $60 M_{\odot}$ star with $v_{\text {ini }}=0 \mathrm{~km} \mathrm{~s}^{-1}$ and $Z=$ $10^{-8}$. The four panels shows the chemical composition at four different stages at the end of the core He-burning phase: Panel a) $Y_{\mathrm{c}}=0.12, M=$ $59.74 M_{\odot}$. b) $Y_{\mathrm{c}}=0.06, M=59.74 M_{\odot}$. c) $Y_{\mathrm{c}}=0.03, M=59.73 M_{\odot}$. d) $Y_{\mathrm{c}}=0.0, M=59.73 M_{\odot}$. The surface metallicity is equal to $10^{-8}$ at the four evolutionary stages.

of magnesium are produced by the reactions ${ }^{22} \mathrm{Ne}(\alpha, \gamma)^{26} \mathrm{Mg}$ and ${ }^{22} \mathrm{Ne}(\alpha, n)^{25} \mathrm{Mg}$. Their high abundances also result from primary nitrogen diffusion into the He-core.

The CO-core mass (cf. Table 3 ) in the rotating model is slightly smaller than in the non-rotating one. This contrasts to what happens at higher metallicity, where rotation tends to increase the CO-core mass (see Hirschi et al. 2004). Again, this results from the mechanism of primary nitrogen production, which induces a large convective zone associated to the H-burning shell, which then prevents this shell from migrating outwards and, thus, the $\mathrm{CO}$ core from growing in mass. Let us recall that in rotating models at solar metallicity, there is no primary nitrogen production due to the less efficient mixing at higher metallicity (see Meynet \& Maeder 2002); thus there is no increase in the H-burning shell activity.

In panel b of Fig. 6, as explained in the previous section, one sees the outer convective zone extending inwards and bringing $\mathrm{CNO}$ elements to the surface. In panels $\mathrm{c}$ and d, massloss efficiently removes these outer layers. At the corresponding stages in the non-rotating model, the outer envelope is not enriched in heavy elements and keeps its mass.

At the end of the He-burning phase (see panels d), the abundance of ${ }^{12} \mathrm{C}$ is significantly smaller in the rotating model than in the non-rotating one. At the same time, the abundances of ${ }^{20} \mathrm{Ne}$ and ${ }^{24} \mathrm{Mg}$ are significantly greater. This is a consequence of helium diffusion into the He-core at the end of the He-burning phase. Let us recall that ${ }^{12} \mathrm{C}$ is destroyed by alpha capture (to produce ${ }^{16} \mathrm{O}$ ), while ${ }^{20} \mathrm{Ne}$ and ${ }^{24} \mathrm{Mg}$ are produced by alpha captures on, respectively, ${ }^{16} \mathrm{O}$ and ${ }^{20} \mathrm{Ne}$. For what concerns the other isotopes of neon and magnesium, one sees that in the rotating models, much higher abundances of ${ }^{25} \mathrm{Mg}$ and ${ }^{26} \mathrm{Mg}$ are reached due to the transformation of the ${ }^{22} \mathrm{Ne}$ at 
Table 3. Helium-, CO-core mass and mass of the remnants (respectively $m_{\alpha}, m_{\mathrm{CO}}$, and $m_{\mathrm{rem}}$ ) of $60 M_{\odot}$ stellar models with and without rotation at $Z=10^{-8}$. The total mass ejected $\left(m_{\mathrm{ej}}\right)$ and the mass ejected of various chemical species $\left(m\left(X_{i}\right)\right)$ are given in solar masses. The values of some isotope ratios (in mass fractions) are also indicated. The case of matter ejected by stellar winds only is distinguished from the case of matter ejected by both the stellar winds and the supernova explosion.

\begin{tabular}{|c|c|c|c|c|}
\hline & $\begin{array}{r}M_{\mathrm{ini}} / \Lambda \\
\\
60\end{array}$ & $\begin{array}{c}Z \begin{array}{c}v_{\text {ini }} \\
{\left[\mathrm{km} \mathrm{s}^{-1}\right]}\end{array} \\
10^{-8} \quad 0\end{array}$ & $\begin{array}{c}M_{\mathrm{ini}} / / \\
60\end{array}$ & $\begin{array}{c}Z \begin{array}{c}v_{\text {ini }} \\
{\left[\mathrm{km} \mathrm{s}^{-1}\right]}\end{array} \\
10^{-8} 800\end{array}$ \\
\hline$m_{\alpha}$ & & 3.08 & & 3.83 \\
\hline$m_{\mathrm{CO}}$ & & 1.61 & & 8.04 \\
\hline$m_{\mathrm{rem}}$ & & 6.65 & & 5.56 \\
\hline & Ма & ejected & $\mathrm{Ma}$ & ejected \\
\hline & WIND & $\mathrm{SN}+\mathrm{WIND}$ & WIND & SN+WIND \\
\hline$m_{\mathrm{ej}}$ & 0.28 & 53.35 & 36.17 & 54.44 \\
\hline$m\left({ }^{4} \mathrm{He}\right)$ & $6.62 \mathrm{e}-02$ & 19.58 & 21.46 & 23.85 \\
\hline$m\left({ }^{12} \mathrm{C}\right)$ & $2.08 \mathrm{e}-10$ & 2.066 & $4.78 \mathrm{e}-03$ & $4.26 \mathrm{e}-01$ \\
\hline$m\left({ }^{13} \mathrm{C}\right)$ & $2.84 \mathrm{e}-12$ & $5.84 \mathrm{e}-09$ & $1.25 \mathrm{e}-03$ & $1.37 \mathrm{e}-03$ \\
\hline$m\left({ }^{14} \mathrm{~N}\right)$ & $6.44 \mathrm{e}-11$ & $1.90 \mathrm{e}-07$ & $1.97 \mathrm{e}-01$ & $2.20 \mathrm{e}-01$ \\
\hline$m\left({ }^{16} \mathrm{O}\right)$ & $1.85 \mathrm{e}-09$ & 12.61 & $6.08 \mathrm{e}-03$ & 13.54 \\
\hline$m\left({ }^{17} \mathrm{O}\right)$ & $8.27 \mathrm{e}-13$ & $6.39 \mathrm{e}-10$ & $7.50 \mathrm{e}-06$ & $8.60 \mathrm{e}-06$ \\
\hline$m\left({ }^{18} \mathrm{O}\right)$ & $4.14 \mathrm{e}-12$ & $6.53 e-10$ & $1.58 \mathrm{e}-08$ & $4.44 \mathrm{e}-03$ \\
\hline \multirow[b]{2}{*}{${ }^{12} \mathrm{C} /{ }^{13} \mathrm{C}$} & \multicolumn{2}{|c|}{ Isotopic ratios } & \multicolumn{2}{|c|}{ Isotopic ratios } \\
\hline & 73.24 & $3.54 \mathrm{e}+08$ & 3.82 & 311 \\
\hline $\mathrm{N} / \mathrm{C}$ & 0.31 & $9.20 \mathrm{e}-08$ & 41.2 & 0.52 \\
\hline $\mathrm{N} / \mathrm{O}$ & 0.03 & $1.51 \mathrm{e}-08$ & 32.4 & 0.02 \\
\hline
\end{tabular}

the end of the core helium-burning phase. The neutrons liberated by the ${ }^{22} \mathrm{Ne}(\alpha, n)^{25} \mathrm{Mg}$ reaction can be captured by iron peak elements, producing some amount of s-process elements (see e.g. Baraffe et al. 1992). In view of the important changes to the interior chemical composition due to rotation, there is good chance that the s-process in the present rotating massive star models is quite different from the one obtained in nonrotating models. This will be examined in later papers.

\section{Chemical composition of the winds and of the supernova ejecta}

\subsection{Wind composition}

Let us first discuss the chemical composition of the winds. The total mass lost, as well as the quantities of various chemical elements ejected by stellar winds, are given in Tables 3 and 4 . The models at $Z=10^{-5}$ were computed with an extended nuclear reaction network including the $\mathrm{Ne}-\mathrm{Na}$ and $\mathrm{Mg}-\mathrm{Al}$ chains, which is why in Table 4 the wind-ejected masses of these elements can be indicated. The stellar yields - i.e., the mass of an isotope newly synthesized and ejected by the star - can be obtained by subtracting the mass of the isotope initially
Table 4. Same as Table 3 for rotating stellar models at $Z=10^{-5}$. The two $60 M_{\odot}$ models were computed with different physical ingredients, see text.

\begin{tabular}{|c|c|c|c|c|}
\hline & \multicolumn{2}{|c|}{$\begin{array}{cccc}M_{\mathrm{ini}} / M_{\odot} Z & \begin{array}{c}v_{\text {ini }} \\
{\left[\mathrm{km} \mathrm{s}^{-1}\right]}\end{array} \\
60 & 10^{-5} & 800\end{array}$} & \multicolumn{2}{|c|}{$\begin{array}{ccc}M_{\mathrm{ini}} / M_{\odot} & Z & v_{\mathrm{ini}} \\
& {\left[\mathrm{km} \mathrm{s}^{-1}\right]}\end{array}$} \\
\hline$m_{\alpha}$ & \multicolumn{2}{|r|}{36.90} & \multicolumn{2}{|c|}{30.69} \\
\hline$m_{\mathrm{CO}}$ & \multicolumn{2}{|r|}{28.60} & \multicolumn{2}{|c|}{27.95} \\
\hline$m_{\mathrm{rem}}$ & \multicolumn{2}{|r|}{8.69} & \multicolumn{2}{|r|}{8.50} \\
\hline & \multicolumn{2}{|c|}{ Mass ejected } & \multicolumn{2}{|c|}{ Mass ejected } \\
\hline & WIND & $\mathrm{SN}+\mathrm{WIND}$ & WIND & SN+WIND \\
\hline$m_{\mathrm{ej}}$ & 23.10 & 51.31 & 29.31 & 51.50 \\
\hline$m\left({ }^{4} \mathrm{He}\right)$ & 12.20 & 18.99 & 12.60 & 14.58 \\
\hline$m\left({ }^{12} \mathrm{C}\right)$ & $3.34 \mathrm{e}-04$ & $5.84 \mathrm{e}-01$ & $1.45 \mathrm{e}-02$ & 2.46 \\
\hline$m\left({ }^{13} \mathrm{C}\right)$ & $6.81 \mathrm{e}-05$ & $1.47 \mathrm{e}-04$ & $3.81 \mathrm{e}-03$ & $2.58 \mathrm{e}-02$ \\
\hline$m\left({ }^{14} \mathrm{~N}\right)$ & $9.78 \mathrm{e}-03$ & $2.51 \mathrm{e}-02$ & $4.29 \mathrm{e}-02$ & $1.87 \mathrm{e}-01$ \\
\hline$m\left({ }^{15} \mathrm{~N}\right)$ & $3.21 \mathrm{e}-07$ & $2.15 \mathrm{e}-06$ & $1.55 \mathrm{e}-06$ & $1.68 \mathrm{e}-05$ \\
\hline$m\left({ }^{16} \mathrm{O}\right)$ & $2.72 \mathrm{e}-04$ & 18.12 & $3.29 \mathrm{e}-02$ & 17.32 \\
\hline$m\left({ }^{17} \mathrm{O}\right)$ & $4.59 \mathrm{e}-07$ & $2.40 \mathrm{e}-06$ & $2.78 \mathrm{e}-05$ & $1.32 \mathrm{e}-04$ \\
\hline$m\left({ }^{18} \mathrm{O}\right)$ & $2.82 \mathrm{e}-08$ & $1.34 \mathrm{e}-03$ & $1.63 \mathrm{e}-08$ & $2.07 \mathrm{e}-04$ \\
\hline$m\left({ }^{19} \mathrm{~F}\right)$ & \multicolumn{2}{|l|}{$1.95 \mathrm{e}-09$} & \multicolumn{2}{|l|}{$1.10 \mathrm{e}-08$} \\
\hline$m\left({ }^{20} \mathrm{Ne}\right)$ & \multicolumn{2}{|l|}{$7.64 \mathrm{e}-06$} & \multicolumn{2}{|l|}{$1.29 \mathrm{e}-05$} \\
\hline$m\left({ }^{21} \mathrm{Ne}\right)$ & \multicolumn{2}{|l|}{$1.84 \mathrm{e}-08$} & \multicolumn{2}{|l|}{$6.99 \mathrm{e}-08$} \\
\hline$m\left({ }^{22} \mathrm{Ne}\right)$ & \multicolumn{2}{|l|}{$4.80 \mathrm{e}-07$} & \multicolumn{2}{|l|}{$3.35 \mathrm{e}-05$} \\
\hline$m\left({ }^{23} \mathrm{Na}\right)$ & \multicolumn{2}{|l|}{$1.22 \mathrm{e}-06$} & \multicolumn{2}{|l|}{$5.61 \mathrm{e}-06$} \\
\hline$m\left({ }^{24} \mathrm{Mg}\right)$ & \multicolumn{2}{|l|}{$4.41 \mathrm{e}-06$} & \multicolumn{2}{|l|}{$6.21 \mathrm{e}-06$} \\
\hline$m\left({ }^{25} \mathrm{Mg}\right)$ & \multicolumn{2}{|l|}{$3.95 \mathrm{e}-07$} & \multicolumn{2}{|l|}{$6.96 \mathrm{e}-07$} \\
\hline$m\left({ }^{26} \mathrm{Mg}\right)$ & \multicolumn{2}{|l|}{$5.68 \mathrm{e}-07$} & \multicolumn{2}{|l|}{$3.07 \mathrm{e}-06$} \\
\hline$m\left({ }^{27} \mathrm{Al}\right)$ & \multicolumn{2}{|l|}{$5.49 \mathrm{e}-06$} & \multicolumn{2}{|l|}{$7.75 \mathrm{e}-06$} \\
\hline & \multicolumn{2}{|c|}{ Isotopic ratios } & \multicolumn{2}{|c|}{ Isotopic ratios } \\
\hline${ }^{12} \mathrm{C} /{ }^{13} \mathrm{C}$ & 4.90 & 3970 & 3.81 & 95.3 \\
\hline $\mathrm{N} / \mathrm{C}$ & 29.3 & 0.04 & 2.96 & 0.08 \\
\hline $\mathrm{N} / \mathrm{O}$ & 36.0 & 0.001 & 1.30 & 0.01 \\
\hline
\end{tabular}

present in that part of the star $^{1}$ from the ejected masses given in Tables 3 and 4.

According to Table 3, the non-rotating model ejects only half a percent of its total mass through stellar winds, which is completely negligible. Moreover, this material has exactly the same chemical composition as does the protostellar cloud from which the star formed. If, at the end of its lifetime, all the stellar material is swallowed by the black-hole resulting from the star collapse, the nucleosynthetic contribution of such stars would be zero. In contrast, the rotating models lose more than $60 \%$ of their initial mass through stellar winds. This material is strongly enriched in CNO elements. Even if all the final stellar mass is engulfed into a black-hole at the end of

1 This quantity may be obtained by multiplying the initial abundance of the isotope considered (given in Table 1) by $m_{\mathrm{ej}}$. 
the evolution, the nucleosynthetic contribution of such stars remains quite significant. As already noted above, the corresponding model at $Z=10^{-5}$ loses less mass by stellar winds (see Table 4). However, the amounts of mass lost remain large and they present strong enrichments in CNO elements, as in the case of the $Z=10^{-8}$ rotating model. Also ${ }^{23} \mathrm{Na}$ and ${ }^{27} \mathrm{Al}$ are somewhat enhanced in the wind material.

Other striking differences between the rotating and nonrotating models concern the ${ }^{12} \mathrm{C} /{ }^{13} \mathrm{C}, \mathrm{N} / \mathrm{C}$, and $\mathrm{N} / \mathrm{O}$ ratios (see Tables 3 and 4). The wind of the non-rotating model shows solar ratios $\left({ }^{12} \mathrm{C} /{ }^{13} \mathrm{C}=73, \mathrm{~N} / \mathrm{C}=0.31\right.$, and $\mathrm{N} / \mathrm{O}=0.03$ in mass fractions). The wind of rotating models is characterised by very low ${ }^{12} \mathrm{C} /{ }^{13} \mathrm{C}$ ratios, around $4-5$ (close to the equilibrium value of the $\mathrm{CN}$ cycle) and by very high $\mathrm{N} / \mathrm{C}$ (between about 3 and 40) and N/O ratios (between 1 and 36). Thus wind material presents the signature of heavily $\mathrm{CNO}$-processed material.

\subsection{Total ejecta composition (wind and supernova ejecta)}

In order to estimate the quantity of mass lost at the time of the supernova explosion (if any), it is necessary to know the mass of the remnant. This quantity is estimated with the relation of Arnett (1991) between the mass of the remnant and the mass of the carbon-oxygen core. The masses of the different elements ejected are then simply obtained by integrating their abundance in the final model between $m_{\text {rem }}$ (see Tables 3 and 4) and the surface. Since the evolution of the present models was stopped before the presupernova stage was reached, the masses of ${ }^{12} \mathrm{C}$ and ${ }^{16} \mathrm{O}$ obtained here might still be somewhat modified by the more advanced nuclear phases.

How does the contribution of the two models (rotating and non-rotating) at $Z=10^{-8}$ compare when both the wind and the supernova contribute to the ejection of the stellar material? First, one sees that the total mass ejected (through winds and supernova explosion) is very similar (on the order of 54-55 $M_{\odot}$ ), due to the fact that the two models have similar CO core masses. Second, one sees that the amount of ${ }^{4} \mathrm{He}$ ejected by the rotating model is slightly higher, whereas the amount of ${ }^{12} \mathrm{C}$ is lower due to the effect discussed above ( $\alpha$-captures at the end of the core He-burning phase). The quantity of ${ }^{16} \mathrm{O}$ ejected is similar in both models. Third, the most important differences between the rotating and non-rotating models occur for ${ }^{13} \mathrm{C},{ }^{14} \mathrm{~N},{ }^{17} \mathrm{O}$, and ${ }^{18} \mathrm{O}$. The abundances of these isotopes are increased by factors between $10^{4}-10^{7}$ in the ejecta of the rotating model. The first three isotopes are produced in the H-burning shell (CNO cycle) and are mainly ejected by the winds, while the last one, produced at the interface between the CO-core and the He-burning shell, is ejected at the time of the supernova explosion. Fourth, rotation also deeply affects the ratios of light elements in the ejected material (see Tables 3 and 4). The effects of rotation are qualitatively similar to those obtained when comparing the composition of the wind material of rotating and non-rotating stellar models. Rotation decreases the ${ }^{12} \mathrm{C} /{ }^{13} \mathrm{C}$ ratios from $3.5 \times 10^{8}$ in the non-rotating case to 311 in the rotating case, while it increases the N/C and N/O ratios, which have values of $\sim 10^{-7}$ and $10^{-8}$ respectively, when $v_{\text {ini }}=$ $0 \mathrm{~km} \mathrm{~s}^{-1}$, and of 0.5 and 0.02 when $v_{\text {ini }}=800 \mathrm{~km} \mathrm{~s}^{-1}$.

In the ejecta of rotating models, composed of both wind and supernova material, the ${ }^{12} \mathrm{C} /{ }^{13} \mathrm{C}$ ratio is higher than in pure wind material, and the $\mathrm{N} / \mathrm{C}$ and $\mathrm{N} / \mathrm{O}$ ratios are smaller. This comes from the fact that the supernova ejecta are rich in helium-burning products characterized by a very high ${ }^{12} \mathrm{C} /{ }^{13} \mathrm{C}$ ratio and by very low $\mathrm{N} / \mathrm{C}$ and $\mathrm{N} / \mathrm{O}$ ratios.

At this point we can ask if the rotating star had lost no mass through stellar winds, and if all the stellar material were ejected at the time of the supernova explosion, would the composition of the ejecta be different with respect to the case discussed above, where part of the material is ejected by the winds and part by the supernova explosion. Let us recall that stellar winds remove layers from the stars at an earlier evolutionary stage than do supernova explosions. If some of these layers, instead of being ejected by the winds, had remained locked inside the star, they would have been processed further by the nuclear reactions. Thus their composition at the end of the stellar evolution would be different from the one obtained if they had been ejected at an earlier time by the winds. Obviously for such differences to be important, mass loss must remove the layers at a sufficiently early time. If it does so only at the very end of the evolution, there would be no chance for the layers to be processed much by the nuclear reactions, and there would be no significant difference whether the mass were ejected by the winds or by the supernova explosion. Actually, this is what happens in our rotating models. As indicated above, the mass is removed at the very end of the He-burning phase, and only material from the H-rich envelope is ejected. Thus, if this material were ejected only at the time of the supernova explosion, it would have kept the same chemical composition as the one in Table 3.

As a result, the chemical composition of the ejecta (wind and supernova) does not depend much on the mass loss, but is deeply affected by rotation. However, the stellar winds may of course be of primary importance if the whole final mass of the star is swallowed in a black-hole at the end of the evolution. In that case, the star will contribute to the interstellar enrichment only by its winds.

Comparing the data given in the right part of Table 3 with the left part of Table 4, one can see the effect produced by an increase in the initial metallicity by three orders of magnitude (all other things being equal). Interestingly, we see that the differences between the two models are in general much smaller than those between the rotating and non-rotating model at a given metallicity. The total ejected mass, and the masses of ${ }^{4} \mathrm{He},{ }^{12} \mathrm{C}$, ${ }^{16} \mathrm{O}$ are similar within factors between 0.8 and 1.4. The quantities of ${ }^{14} \mathrm{~N}$ and ${ }^{13} \mathrm{C}$ are within an order of magnitude, and the masses of ${ }^{17} \mathrm{O}$ and ${ }^{18} \mathrm{O}$ differ by a factor 3 . We are thus far from the factors $10^{4}-10^{7}$ between the results of the rotating and non-rotating models at $Z=10^{-8}$ ! The effects of rotation at extremely low metallicity are much larger than the effects of a change in the initial $Z$ content.

The results given on the right side of Table 4 corresponds to the model described in Meynet et al. (2005). It differs from the present models by the mass loss and mixing prescription (see Sect. 3.2). As already emphasized above, the results are 
qualitatively very similar. However, quantitatively, they present some differences. For instance, the quantity of ${ }^{12} \mathrm{C}$ in the model presented on the right side of Table 4 is larger by a factor 4 compared to the value given on the left side of the same table. The right model presents a smaller helium core, an effect mainly due to higher mass loss rates. This favours larger ejections of carbon by the winds and also by the supernova, since smaller helium cores lead to higher $\mathrm{C} / \mathrm{O}$ ratios at the end of the helium-burning phase. In the right model, the quantity of ${ }^{16} \mathrm{O}$ is decreased by about $4 \%$. The ejected masses of ${ }^{13} \mathrm{C}$ and ${ }^{17} \mathrm{O}$ are increased by factors of 176 and 55, respectively. The masses of the other isotopes differ by less than an order of magnitude.

\section{Link with the extremely metal-poor C-rich stars}

\subsection{Observations and existing interpretations}

Spectroscopic surveys of very metal-poor stars (Beers et al. 1992; Beers 1999; Christlieb 2003) have shown that CEMP stars account for up to about $25 \%$ of stars with metallicities lower than $[\mathrm{Fe} / \mathrm{H}] \sim-2.5$ (see e.g. Lucatello et al. 2004). A star is said to be C-rich if $[\mathrm{C} / \mathrm{Fe}]>1$. A large proportion of these CEMP stars also present enhancements in their neutron capture elements (mainly $s$-process elements). A few of them also appear to exhibit large enhancements in $\mathrm{N}$ and $\mathrm{O}$. The most iron-deficient stars observed so far are CEMP stars. These stars are HE 0107-5240, a giant halo star, and HE 1327-2326, a dwarf or subgiant halo star. The star HE 0107-5240 $([\mathrm{Fe} / \mathrm{H}]=-5.3)$ presents the following CNO surface abundances: $[\mathrm{C} / \mathrm{Fe}]=4.0,[\mathrm{~N} / \mathrm{Fe}]=2.3$, and $[\mathrm{O} / \mathrm{Fe}]=2.4$ (Christlieb et al. 2004; Bessell et al. 2004). The ratio ${ }^{12} \mathrm{C} /{ }^{13} \mathrm{C}$ has also been tentatively estimated by Christlieb et al. (2004), who suggest a value of about 60 , but with a great uncertainty. They can, however, rule out a value inferior to 50 (let us recall that the solar ratio is $\sim 73$ ). The star HE 1327-2326 has $[\mathrm{Fe} / \mathrm{H}]=-5.4$ and $\mathrm{CNO}$ surface abundances: $[\mathrm{C} / \mathrm{Fe}]=4.1$, $[\mathrm{N} / \mathrm{Fe}]=4.5,[\mathrm{O} / \mathrm{Fe}]<4.0($ Frebel et al. 2005).

The origin of the high carbon abundance is still an open question and various scenarios have been proposed:

1. The primordial scenarios: in this case the abundances observed at the surface of CEMP stars are the abundances of the cloud from which the star formed. The protostellar cloud was enriched in carbon by one or a few stars from a previous generation. For instance, Umeda \& Nomoto (2003) propose that the cloud from which HE 0107-5240 formed was enriched by the ejecta of one Pop III $25 M_{\odot}$ star, which had exploded with low explosion energy (on the order of $3 \times 10^{50} \mathrm{erg}$ ) and experienced strong mixing and fallback at the time of the supernova explosion. The mixing is necessary to create the observed high-level enrichments in light elements, and the fallback is necessary to retain a large part of the iron peak elements. Limongi et al. (2003) suggest that the cloud was enriched by the ejecta of two supernovae from progenitors with masses of about 15 and $35 M_{\odot}$.

2. The accretion/mass transfer scenarios: some authors have proposed that this particular abundance pattern results from accretion of interstellar material and from a companion (for instance an AGB star, as proposed by Suda et al. 2004). As far as the nucleosynthetic origin is concerned, this scenario is not fundamentally different from the first one.

3. The in situ scenarios: finally, some authors have explored the possibility that the star itself could have produced the particular abundance pattern seen at its surface (Picardi et al. 2004). The overabundance of nitrogen might easily be explained in the frame of this scenario, if the star had begun its evolution with the high carbon and oxygen overabundance. In fact we did perform a test calculation of a non-rotating $0.8 M_{\odot}$ stellar model at $[\mathrm{Fe} / \mathrm{H}]=-5.3$ with an initial value of $[\mathrm{C} / \mathrm{Fe}]$ and $[\mathrm{O} / \mathrm{Fe}]$ equal to 4.0 and 2.3 , respectively, i.e. equal to the abundances observed at the surface of HE 0107-5240. We found that, when the star reaches the value of the effective temperature $\left(T_{\text {eff }}=5100 \pm\right.$ $150 \mathrm{~K})$ and of gravity $(\log g=2.2 \pm 0.3)$ of HE 0107-5240 (Christlieb et al. 2002), the surface nitrogen enrichment is well within the range of the observed values. However, it appears difficult to invoke similar processes to explain the high carbon and oxygen enhancements (see Picardi et al. 2004).

\subsection{No "in situ" CN production}

An abundance pattern typical of CEMP stars has been observed at the surface of non-evolved stars (Norris et al. 1997; Plez \& Cohen 2005; Frebel et al. 2005). Among the most recent observations, let us mention the subgiant or dwarf star HE 1327-2326 (Frebel et al. 2005, see above) and the dwarf star G77-61 (Plez \& Cohen 2005). The initial mass of G77-61 is estimated to be between 0.3 and $0.5 M_{\odot}$, and it has an $[\mathrm{Fe} / \mathrm{H}]=-4.03,[\mathrm{C} / \mathrm{Fe}]=2.6,[\mathrm{~N} / \mathrm{Fe}]=2.6$, and a ${ }^{12} \mathrm{C} /{ }^{13} \mathrm{C}$ ratio of $5 \pm 1$. In this case, there is no way for the star, which burns its hydrogen through the pp chains, to produce nitrogen. There is even less possibility of producing surface enhancements of carbon and oxygen. Therefore, the "in situ" scenario can be excluded, at least for this star. In that case, only the first and second scenarios are possible. The same is true for explaining the very high overabundances of carbon and nitrogen at the surface of HE 1327-2326.

The values observed at the surface of non-evolved and evolved stars are shown in Fig. 8. In the second case, the surface may have been depleted in carbon and oxygen and enriched in nitrogen due to the dredge-up that occurs along the red giant branch. On the other hand, in the case of the nonevolved stars, as explained above, this mechanism cannot be invoked, and the measured abundances reflect the abundances of the cloud that gave birth to the star. On the whole, the distributions of elements is similar for evolved and non-evolved stars, which favours the primordial scenario.

In the following, we explore the first two scenarios using our fast-rotating models. The abundance pattern observed at the surface of CEMP stars seems to be a mixture of hydrogen and helium burning products. Since rotation allows these products to coexist in the outer layers of stars (both in massive and 


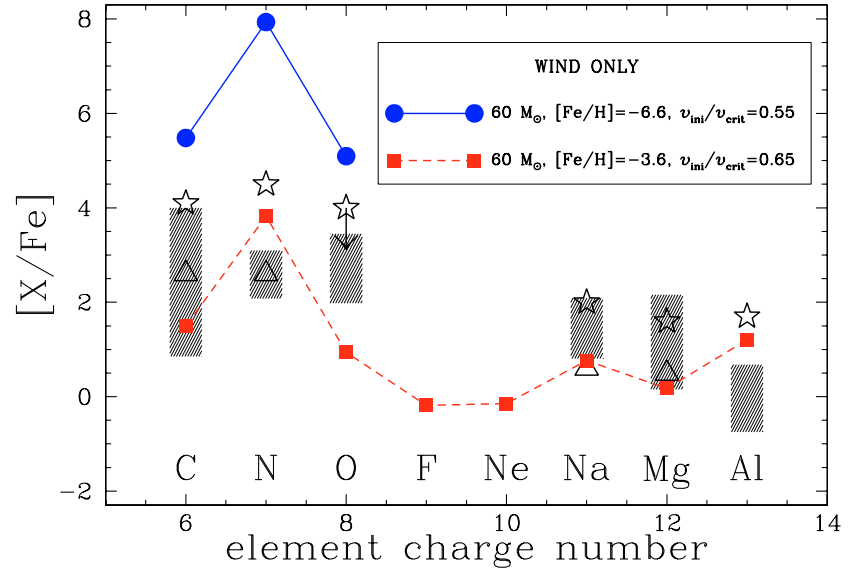

Fig. 8. Chemical composition of the wind of rotating $60 M_{\odot}$ models (solid circles and squares). The hatched areas correspond to the range of values measured at the surface of giant CEMP stars: HE 0107-5240, $[\mathrm{Fe} / \mathrm{H}] \simeq-5.3$ (Christlieb et al. 2004); CS 22949-037, [Fe/H] $\simeq-4.0$ (Norris et al. 2001; Depagne et al. 2002); CS 29498-043, [Fe/H] $\simeq$ -3.5 (Aoki et al. 2004). The empty triangles (Plez \& Cohen 2005, $[\mathrm{Fe} / \mathrm{H}] \simeq-4.0)$ and stars (Frebel et al. 2005, $[\mathrm{Fe} / \mathrm{H}] \simeq-5.4$, only an upper limit is given for $[\mathrm{O} / \mathrm{Fe}]$ ) correspond to non-evolved CEMP stars (see text).

intermediate mass stars), this seems a useful direction for our research.

\subsection{Comparison with wind composition}

Let us first see if the CEMP stars could be formed from material made up of massive star winds, or at least heavily enriched by winds of massive stars. At first sight, such a model might appear quite unrealistic, since the period of strong stellar winds is rapidly followed by the supernova ejection, which would add the ejecta of the supernova itself to the winds. However, for massive stars at the end of their nuclear lifetime, a black hole, that swallows the whole final mass, might be produced. In that case, the massive star would contribute to the local chemical enrichment of the interstellar medium only through its winds. Let us suppose that such a situation has occurred and that the small halo star we observe today was formed from the shock induced by the stellar winds with the interstellar material. What would its chemical composition be? Its iron content would be the same as the iron abundance of the massive star. Indeed, the iron abundance of the interstellar medium would have no time to change much in the brief massive star lifetime, and the massive star wind ejecta are neither depleted nor enriched in iron.

The abundances of the other elements in the stellar winds for our two rotating $60 M_{\odot}$ at $Z=10^{-8}$ and $10^{-5}$ are shown in Fig. 8. The ordinate $[X / \mathrm{Fe}]$ is given by the following expression:

$[X / \mathrm{Fe}]=\log \left(\frac{X}{X_{\odot}}\right)-\log \left(\frac{X(\mathrm{Fe})}{X(\mathrm{Fe})_{\odot}}\right)$,

where $X$ is the mass fraction of element $X$ in the wind ejecta, and $X_{\odot}$ in the Sun. Similarly, the symbols $X(\mathrm{Fe})$ and $X(\mathrm{Fe})_{\odot}$ refer to the mass fraction of ${ }^{56} \mathrm{Fe}$ in the wind material or in the Sun. Here we suppose that $\log \left(X(\mathrm{Fe}) / X(\mathrm{Fe})_{\odot}\right) \sim[\mathrm{Fe} / \mathrm{H}]$, since the mass fraction of hydrogen remains approximately constant, whatever the metallicity between $Z=10^{-8}$ and 0.02 . The values of $[\mathrm{Fe} / \mathrm{H}]$ are those corresponding to the initial metallicity of the models (for $Z=10^{-8}$ one has $\log (X(\mathrm{Fe}) / X(\mathrm{H})$ ) $=$ -9.38 , see Table 1). The solar abundances are those chosen by Christlieb et al. (2004) and Bessell et al. (2004) in their analysis of the star HE 0107-5240, and they correspond to the solar abundances obtained recently by Asplund et al. (2005). In particular, $\log (X(\mathrm{Fe}) / X(\mathrm{H}))_{\odot}=-2.80$, thus $[\mathrm{Fe} / \mathrm{H}]=-6.6$ at $Z=$ $10^{-8}$, and $[\mathrm{Fe} / \mathrm{H}]=-3.6$ at $Z=10^{-5}$.

From Fig. 8, we see that the winds are strongly enriched in CNO elements. The model at $Z=10^{-5}$, computed with an extended nuclear reaction network, allows us to look at the abundances in the winds of heavier elements. The wind material is also somewhat enriched in $\mathrm{Na}$ and $\mathrm{Al}$. Before comparing with the observations, let us first note:

1) The more metal-poor model is shifted toward higher values compared to the metal rich one. If, in both models, the mass fraction of element $X$ were the same in the wind ejecta, then one would expect a shift by 3 dex when the iron content of the model goes from $[\mathrm{Fe} / \mathrm{H}]=-3.6$ to $[\mathrm{Fe} / \mathrm{H}]=-6.6$. The actual shift is approximately 3.6 dex, slightly more than the iron content difference of 3 dex between the two models. The additional 0.6 dex comes from the more efficient mixing in the metal-poorer models.

2) In the frame of the hypotheses made here, i.e. a halo star made from the wind ejecta that triggered its formation, we should compare the wind composition from a massive star model with the same initial iron content as in the halo star we considered. The range of iron contents in the models, $[\mathrm{Fe} / \mathrm{H}]$ equal from -6.6 to -3.6 , covers the range of iron content of the CEMP plotted in Fig. 8, whose $[\mathrm{Fe} / \mathrm{H}]$ are between -5.4 and -3.5 . However, the $[\mathrm{Fe} / \mathrm{H}]=-6.6$ model is well below the lower bound of the observed $[\mathrm{Fe} / \mathrm{H}]$, making this model less interesting for comparisons with the presently available observations. In that respect the $[\mathrm{Fe} / \mathrm{H}]=-3.6$ model, which has an iron content that is comparable to the iron-richest stars observed, is more interesting.

3) Any dilution with some amount of interstellar material would lower the abundance of the element $X$ without changing the mass fraction of iron. In that case the values plotted in Fig. 8 are shifted to lower values, but the relative abundances of the elements will not change (as far as the main source of the elements considered are the wind ejecta).

Keeping in mind these three comments, it appears that what has to be compared with the observations are more the relative abundances between the $\mathrm{CNO}$ elements than the actual values of the $[\mathrm{X} / \mathrm{Fe}]$ ratios, which will depend on the initial metallicity of the model considered, as well as on the dilution factor.

From Fig. 8, and Tables 3 and 4, one sees that, for the two metallicities considered here, the wind material of rotating models is characterised by N/C and N/O ratios between $\sim 1$ and 40 and by ${ }^{12} \mathrm{C} /{ }^{13} \mathrm{C}$ ratios around $4-5$. These values are compatible with the ratios observed at the surface of CS 22949-037 (Depagne et al. 2002): N/C 3 and ${ }^{12} \mathrm{C} /{ }^{13} \mathrm{C} \sim 4$. The observed value for $\mathrm{N} / \mathrm{O}(\sim 0.2)$ is smaller than the range of 
theoretical values, but greater than the solar ratio $(\sim 0.03)$. Thus the observed N/O ratio also bears the mark of some CNO processing, although slightly less pronounced than in our stellar wind models.

On the whole, a stellar wind origin for the material composing this star does not appear out of order in view of these comparisons, all the more so if one considers that, in the present comparison, there is no fine tuning of some parameters in order to obtain the best agreement possible. The theoretical results are directly compared to the observations. Moreover only a small subset of possible initial conditions has been explored.

Other CEMP stars present, however, lower values for the $\mathrm{N} / \mathrm{C}$ and N/O ratios and higher values for the ${ }^{12} \mathrm{C} /{ }^{13} \mathrm{C}$ ratio. For these cases, it appears that the winds of our rotating $60 M_{\odot}$ models appear to be too strongly CNO-processed (too high N/C and $\mathrm{N} / \mathrm{O}$ ratios and too low ${ }^{12} \mathrm{C} /{ }^{13} \mathrm{C}$ ratios). Better agreement would be obtained if the observed abundances also stem from material coming from the $\mathrm{CO}$-core and ejected either by strong late stellar winds or in a supernova explosion.

\subsection{Expression of abundance ratios in total ejecta (winds and supernova)}

To find the initial chemical composition of stars that would form from a mixture of wind and supernova ejecta with interstellar medium material, let us define $X_{\mathrm{ej}}$ as the mass fraction of element $X$ in the ejecta (wind and supernova). This quantity can be obtained from the stellar models and computed according to the expression below:

$X_{\mathrm{ej}}=\frac{X_{\text {wind }} m_{\text {wind }}+X_{\mathrm{SN}} m_{\mathrm{SN}}}{m_{\mathrm{wind}}+m_{\mathrm{SN}}}$,

where $X_{\text {wind }}$ and $X_{\mathrm{SN}}$ are the mass fractions of element $X$ in the wind, in the supernova ejecta. Here $m_{\text {wind }}$ and $m_{\mathrm{SN}}$ are the masses ejected by the stellar winds and at the time of the supernova explosion. To obtain the mass of the remnants, we adopted the relation obtained by Arnett (1991) between the masses of the remnant and the CO core. This method is the same as the one adopted by Maeder (1992).

The total mass ejected by the star, $m_{\mathrm{ej}}=m_{\text {wind }}+m_{\mathrm{SN}}$, is mixed with some amount of interstellar material $m_{\mathrm{ISM}}$. The mass fraction of element $X$ in the material composed from the ejecta mixed with the interstellar medium will be

$X=\frac{X_{\mathrm{ej}} m_{\mathrm{ej}}+X_{\mathrm{ini}} m_{\mathrm{ISM}}}{m_{\mathrm{ej}}+m_{\mathrm{ISM}}}=\frac{X_{\mathrm{ej}} \frac{m_{\mathrm{ej}}}{m_{\mathrm{ISM}}}+X_{\mathrm{ini}}}{\frac{m_{\mathrm{ej}}}{m_{\mathrm{ISM}}}+1}$,

where $X_{\text {ini }}$ is the mass fraction of element $X$ in the interstellar medium. In our case the interstellar medium is very metal poor so that one can consider $X_{\text {ini }} \sim 0$ for the heavy elements synthesised in great quantities by the star (note that this cannot be done for nitrogen ejected by the non-rotating $60 M_{\odot}$ stellar model). We also suppose that $m_{\mathrm{ej}} \ll m_{\mathrm{ISM}}$ and thus $X=\left(X_{\mathrm{ej}} m_{\mathrm{ej}}\right) / m_{\mathrm{ISM}}$. Using these expressions, one can write

$[\mathrm{Fe} / \mathrm{H}]=\log \left(\frac{X(\mathrm{Fe})_{\mathrm{ej}}}{X(\mathrm{Fe})_{\odot}}\right)+\log \left(\frac{m_{\mathrm{ej}}}{m_{\mathrm{ISM}}}\right)$, assuming, as we did above, that $X(\mathrm{H})_{\odot} / X(\mathrm{H})_{\mathrm{ej}} \approx 1$. Values of $[X / \mathrm{Fe}]$ are obtained using the expression

$[X / \mathrm{Fe}]=[X / \mathrm{H}]-[\mathrm{Fe} / \mathrm{H}]=\log \left(\frac{X_{\mathrm{ej}}}{X_{\odot}}\right)-\log \left(\frac{X(\mathrm{Fe})_{\mathrm{ej}}}{X(\mathrm{Fe})_{\odot}}\right)$.

One needs to have an estimate for both $m_{\mathrm{ej}} / m_{\mathrm{ISM}}$ (the dilution factor) and for the mass fraction of iron in the ejecta $X(\mathrm{Fe})_{\mathrm{ej}}$. A precise quantitative determination of $X(\mathrm{Fe})_{\mathrm{ej}}$ and $m_{\mathrm{ej}} / m_{\mathrm{ISM}}$ from theory is quite difficult. For instance, for a given initial mass, the quantity of iron ejected by the supernova can vary by orders of magnitudes depending on the mass cut, the energetics of the supernova, and the geometry of the explosion (see e.g. Maeda \& Nomoto 2003). On the other hand, the dilution factor will depend on the energetics of the supernova, among other parameters. In the absence of any very precise guidelines, we determined the two unknown quantities, the dilution factor, and the mass of ejected iron, requiring that the mixture will have $[\mathrm{Fe} / \mathrm{H}]=-5.4$ and $[\mathrm{O} / \mathrm{Fe}]=+3.5$. The first value corresponds to the values observed at the surface of the star HE 1327-2326 (Frebel et al. 2005), and the second value is below the upper limit of $[\mathrm{O} / \mathrm{Fe}](<4.0)$ found for this star. Doing so, one can write,

$[\mathrm{X} / \mathrm{Fe}]=[\mathrm{X} / \mathrm{O}]+[\mathrm{O} / \mathrm{Fe}]=\log \left(\frac{X_{\mathrm{ej}}}{X_{\odot}}\right)-\log \left(\frac{X(\mathrm{O})_{\mathrm{ej}}}{X(\mathrm{O})_{\odot}}\right)+3.5$,

where $X(\mathrm{O})$ is the mass fraction of oxygen. The mass fraction of ejected iron can be estimated from

$[\mathrm{O} / \mathrm{Fe}]=\log \left(\frac{X(\mathrm{O})_{\mathrm{ej}}}{X(\mathrm{O})_{\odot}}\right)-\log \left(\frac{X(\mathrm{Fe})_{\mathrm{ej}}}{X(\mathrm{Fe})_{\odot}}\right)=3.5$,

and the dilution factor can be obtained from

$[\mathrm{Fe} / \mathrm{H}]=\log \left(\frac{X(\mathrm{Fe})_{\mathrm{ej}}}{X(\mathrm{Fe})_{\odot}}\right)+\log \left(\frac{m_{\mathrm{ej}}}{m_{\mathrm{ISM}}}\right)=-5.4$.

\subsection{Results from the "wind plus supernova ejecta" model}

Using the above formulae, let us now discuss what can be expected for the chemical composition of a very metal-poor star formed from such a mixture. This includes the CNO elements for which the present models can give consistent estimates and the case of our models at $Z=10^{-8}([\mathrm{Fe} / \mathrm{H}]=$ $-6.6)$, which are the models that are compatible with the requirement that the mixture of ejecta and ISM material must have an $[\mathrm{Fe} / \mathrm{H}]=-5.4$. Obviously our second series of models at $Z=10^{-5}([\mathrm{Fe} / \mathrm{H}]=-3.6)$ does not fit this requirement. Imposing $[\mathrm{O} / \mathrm{Fe}]=3.5$ and $[\mathrm{Fe} / \mathrm{H}]=-5.4$ implies masses of iron that are being ejected on the order of $1 \times 10^{-3} M_{\odot}$ and mixed with a mass of interstellar medium of about $2 \times 10^{5} M_{\odot}$. The mass of ejected iron (actually in the form of ${ }^{56} \mathrm{Ni}$ ) is very small compared to the classical values of $0.07-0.10 M_{\odot}$. On the other hand, this quantity can be very small if a large part of the mass falls back onto the remnant (Umeda \& Nomoto 2003). The mass of interstellar gas collected by the shock wave can be related to the explosion energy $E_{\text {exp }}$ through (see Shigeyama \& Tsujimoto 1998)

$M_{\mathrm{ISM}}=5.1 \times 10^{4} M_{\odot}\left(\frac{E_{\mathrm{exp}}}{10^{51} \mathrm{erg}}\right)$. 


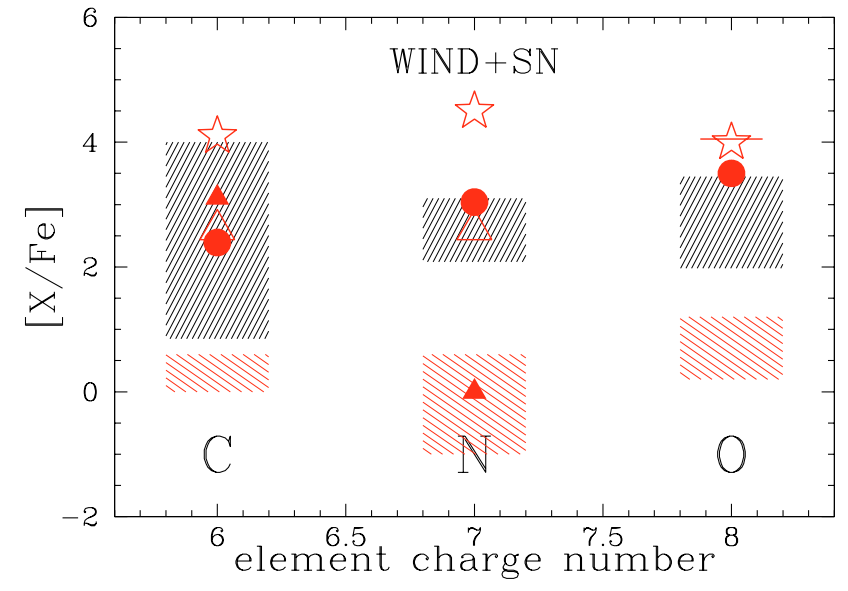

Fig. 9. Chemical composition of the ejecta (wind and supernova) of $60 M_{\odot}$ models: solid circles and triangles correspond to models at $Z=10^{-8}([\mathrm{Fe} / \mathrm{H}]=-6.6)$ with and without rotation. The $[\mathrm{N} / \mathrm{Fe}] \mathrm{ra}-$ tio for the non-rotating model is equal to 0 , i.e., no $\mathrm{N}$-enrichment is expected. The hatched areas (moving from top right down to the left) correspond to the range of values measured at the surface of giant CEMP stars (same stars as in Fig. 8). The empty triangles (Plez $\&$ Cohen 2005) and stars (Frebel et al. 2005, only an upper limit is given for $[\mathrm{O} / \mathrm{Fe}]$ ) correspond to non-evolved CEMP stars (see text). The hatched areas ( $\mathrm{L}$ to $\mathrm{R}$ from top) show the range of values measured at the surface of normal halo giant stars by Cayrel et al. (2004) and Spite et al. (2005, unmixed sample only, see text).

A mass of $2 \times 10^{5} M_{\odot}$ would correspond to an energy equal to $4 \times 10^{51} \mathrm{erg}$, i.e., a value well in the range of energies released by supernova explosion. Thus imposing $[\mathrm{O} / \mathrm{Fe}]=3.5$ and $[\mathrm{Fe} / \mathrm{H}]=-5.4$ does not imply unrealistic values for the mass of iron that is ejected and for the mass of interstellar medium swept up by the shock wave of the supernova explosion.

The theoretical ratios for the $\mathrm{CNO}$ elements are shown in Fig. 9 and compared with the ratios observed at the surface of CEMP stars and of normal giant halo stars by Cayrel et al. (2004) and Spite et al. (2005). For oxygen, the value of 3.5 is obtained by construction, so it does not provide any constraint; however, see the previous paragraph. More interesting, of course, are the carbon and nitrogen abundances. One sees that both the non-rotating and rotating models might account for some level of C-enrichment that is compatible with the range of values observed at the surface of CEMP stars. However, only the rotating models produce $\mathrm{N}$-rich material at this level. Figure 9 also shows that the predicted value for the $\mathrm{N} / \mathrm{C}$ and $\mathrm{N} / \mathrm{O}$ ratios from the rotating model appear to agree with the observed values of these ratios at the surface of CEMP stars. Thus as expected, the addition of material from the $\mathrm{CO}$ core (here ejected at the time of the supernova explosion) to the wind material (mainly enriched in CNO-processed material), reduces the $\mathrm{N} / \mathrm{C}$ and $\mathrm{N} / \mathrm{O}$ ratios.

The theoretical values in the wind-plus-supernova ejecta model for the ratio ${ }^{12} \mathrm{C} /{ }^{13} \mathrm{C}$ are between 100 and 4000 for the rotating models (see Tables 3 and 4). The value predicted by the non-rotating model is much higher, on the order of $10^{8}$. Compared to the observed values, which are between 4 and 60, the value of the non-rotating model is in excess by at least seven orders of magnitude. The situation is much more favourable for the rotating models. In this last case, the predicted values are still somewhat too high, but by much lower factors.

Other proportions probably exist between wind and supernova mass ejecta than those considered here, which would provide a better fit to the observed surface abundances of CEMP stars. Also more models need to be calculated for exploring the set of initial parameters leading to a good correspondence between theory and observations. From the large range of results obtained by different initial conditions, there is little doubt, that such a given set of parameters exists. The ${ }^{12} \mathrm{C} /{ }^{13} \mathrm{C}$ ratio appears extremely sensitive to input parameters, so it may be a powerful tool for a closer identification of the exact nucleosynthetic site.

As can be seen from Fig. 9, the abundances observed at the surface of the normal giant stars by Cayrel et al. (2004) and Spite et al. (2005) are not far from solar ratios, and are well below the range of values observed at the surface of CEMP stars. Only the subset of stars qualified as unmixed by Spite et al. (2005), i.e., presenting no evidence of $\mathrm{C}$ to $\mathrm{N}$ conversion, has been plotted here. Probably these stars are formed from a reservoir of matter made up of the ejecta of different initial mass stars, convolved with a proper distribution of the initial rotation velocities, while the C-rich stars require some special circumstances involving a few or maybe only one nucleosynthetic event.

\subsection{Chemical composition of the envelopes of $E-A G B$ stars}

One can wonder whether intermediate mass stars could also play a role in explaining the peculiar abundance pattern of CEMP stars. For instance, Suda et al. (2004) propose that the small halo star, observed today as a CEMP star, was the secondary in a close binary system. The secondary might have accreted matter from its evolved companion, an AGB star, and might have thus acquired at least part of its peculiar surface abundance pattern.

The physical conditions encountered in the advanced phases of an intermediate mass star are not so different from the one realised in massive stars. Thus the same nuclear reaction chains can occur and lead to similar nucleosynthetic products. Also the lifetimes of massive stars (on the order of a few million years) are not very different from the lifetimes of the most massive intermediate mass stars; typically a $7 M_{\odot}$ has a lifetime on the order of $40 \mathrm{Myr}$, only an order of magnitude higher than a $60 M_{\odot}$ star. Moreover, the observation of s-process element overabundances at the surface of some CEMP stars also point toward a possible Asymptotic Giant Branch (AGB) star origin ${ }^{2}$ for the material composing the CEMP stars.

To explore this scenario, we computed a $7 M_{\odot}$ with $v_{\text {ini }}=$ $800 \mathrm{~km} \mathrm{~s}^{-1}$ at $Z=10^{-5}$, and with the same physical ingredients as the $60 M_{\odot}$ stellar models of the present paper.

\footnotetext{
2 Note that massive stars also produce s-process elements. The massive star s-process elements have low atomic mass number (A inferior to about 90) and are known under the name of the weak component of the s-process.
} 


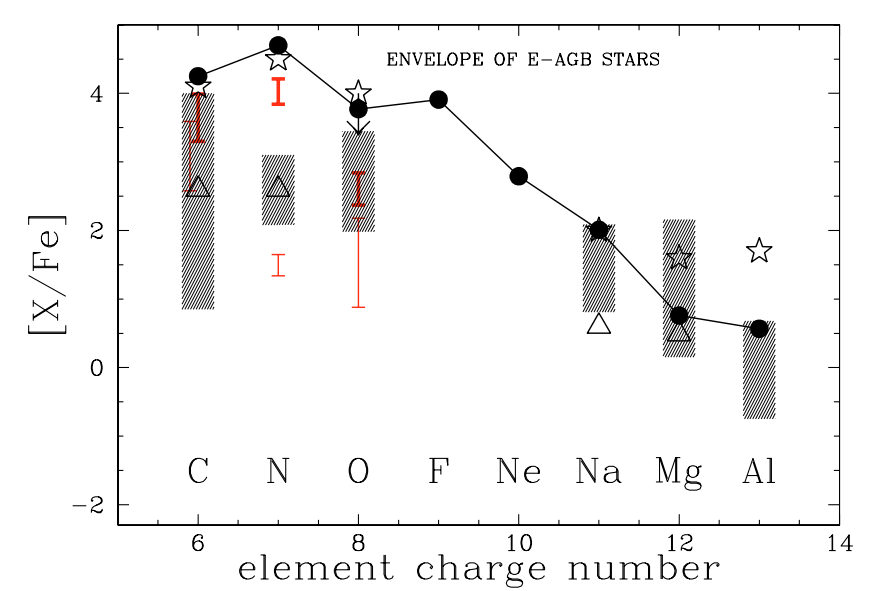

Fig. 10. Chemical composition of the envelopes of E-AGB stars compared to abundances observed at the surface of CEMP stars (hatched areas). The continuous line shows the case of a $7 M_{\odot}$ at $Z=10^{-5}$ $([\mathrm{Fe} / \mathrm{H}]=-3.6)$ with $v_{\text {ini }}=800 \mathrm{~km} \mathrm{~s}^{-1}$. The vertical lines (shown as "error bars") indicate the ranges of values for CNO elements in the stellar models of Meynet \& Maeder (2002) (models with initial masses between 2 and $7 M_{\odot}$ at $Z=10^{-5}$ ). The thick and thin lines correspond to rotating $\left(v_{\text {ini }}=300 \mathrm{~km} \mathrm{~s}^{-1}\right)$ and non-rotating models. The empty triangles (Plez \& Cohen 2005) and stars (Frebel et al. 2005, only an upper limit is given for $[\mathrm{O} / \mathrm{Fe}]$ ) correspond to non-evolved CEMP stars.

In contrast to the $60 M_{\odot}$ models, the $7 M_{\odot}$ stellar model loses little mass during the core $\mathrm{H}$ - and He-burning phase, so that the star has still nearly its whole original mass at the early asymptotic giant branch stage (the actual mass at this stage is $\left.6.988 M_{\odot}\right)$. This is because the star never reaches the breakup limit during the MS phase; and, due to rotational mixing and dredge-up, the metallicity enhancement at the surface only occurs very late, when the star evolves toward the red part of the HR diagram after the end of the core He-burning phase. At this stage, the outer envelope of the star is enriched in primary CNO elements, and the surface metallicity reaches about 1000 times the initial metallicity. If such a star is in a close binary system, there is good chance that mass transfer occurs during this phase of expansion of the outer layers. In that case, the secondary may accrete part of the envelope of the E-AGB star.

From the $7 M_{\odot}$ stellar model, we can estimate the chemical composition of the envelope at the beginning of the thermal pulse AGB phase. Here we call envelope all the material above the $\mathrm{CO}$-core. The result is shown in Fig. 10 (continuous line with solid circles). We also plotted the values obtained from the models of Meynet \& Maeder (2002) for initial masses between 2 and $7 M_{\odot}$ at $Z=10^{-5}$ and with $v_{\text {ini }}=0$ and $300 \mathrm{~km} \mathrm{~s}^{-1}$.

Before discussing the comparisons with observations, let us make two remarks: 1) as was the case for the theoretical predictions of the massive star winds, the values given here have not been adjusted to fit the observed values but result from the basic physics of the models; 2) the initial metallicity of our AGB models $([\mathrm{Fe} / \mathrm{H}]=-3.6)$ is at the higher range of values of the metallicities observed for the CEMP stars. However, based on the results from our massive star models at $[\mathrm{Fe} / \mathrm{H}]=-6.6$ and -3.6 (see Fig. 8), we see that the overall pattern of the abundances will probably remain quite similar for a lower initial metallicity. Only a shift toward higher values along the vertical axis is expected when the initial metallicity of the model is decreased.

Looking at Fig. 10, one can note the three following points:

1. The envelope of rotating intermediate mass stars presents a chemical composition in carbon, nitrogen, and oxygen that agrees well with the observed value at the surface of CEMP stars. In particular, compared to the wind material of massive stars (see Fig. 8), the N/C ratios and N/O ratios are in better agreement. The non-rotating models cannot account for the high overabundances in nitrogen and oxygen.

2. The ${ }^{12} \mathrm{C} /{ }^{13} \mathrm{C}$ ratios in our rotating models are between 19 and 2500, with the lowest values corresponding to the most massive intermediate-mass star models. The non-rotating models give values between $3 \times 10^{5}$ and $2 \times 10^{6}$. Again here, rotating models agree much better with the observed values, although very low ${ }^{12} \mathrm{C} /{ }^{13} \mathrm{C}$ values (on the order of $4-5$, as observed e.g. at the surface of the dwarf halo star G77 61, see Plez \& Cohen 2005) seem to be reproduced only by massive star models (wind material only).

3. For sodium and aluminum, the ratios predicted by our $7 M_{\odot}$ model with $v_{\text {ini }}=800 \mathrm{~km} \mathrm{~s}^{-1}$ fit the observed values well. In the case of magnesium, good agreement is also obtained.

Thus we see that the envelopes of AGB stellar models with rotation show a very similar chemical composition to the one observed at the surface of CEMP stars. It is, however, still difficult to say that rotating intermediate mass star models are better than rotating massive star models in this respect. Probably, some CEMP stars are formed from massive star ejecta and others from AGB star envelopes. Interestingly at this stage, some possible ways to distinguish between massive star wind material and AGB envelopes do appear. Indeed, we just saw above that massive star wind material is characterised by a very low ${ }^{12} \mathrm{C} /{ }^{13} \mathrm{C}$ ratio, while intermediate mass stars seem to present higher values for this ratio. The AGB envelopes would also present very high overabundances of ${ }^{17} \mathrm{O},{ }^{18} \mathrm{O},{ }^{19} \mathrm{~F}$, and ${ }^{22} \mathrm{Ne}$, while wind of massive rotating stars present a weaker overabundance of ${ }^{17} \mathrm{O}$ and depletion of ${ }^{18} \mathrm{O},{ }^{19} \mathrm{~F}$, and ${ }^{22} \mathrm{Ne}$. As discussed in Frebel et al. (2005), the ratio of heavy elements, such as the strontium-to-barium ratio, can also give clues to the origin of the material from which the star formed. In the case of HE 1327-2326, Frebel et al. (2005) give a lower limit of $[\mathrm{Sr} / \mathrm{Ba}]>-0.4$, which suggests that strontium was not produced in the main s-process occurring in AGB stars, thus leaving the massive star hypothesis as the best option, in agreement with the result from ${ }^{12} \mathrm{C} /{ }^{13} \mathrm{C}$ in G77-61 (Plez \& Cohen 2005) and CS 22949-037 (Depagne et al. 2002).

\section{Conclusion}

We have proposed a new scenario for the evolution of very metal-poor massive stars. This scenario requires no new physical processes, as it is based on models that have been extensively compared to observations of stars at solar composition and in the LMC and SMC. The changes with respect to classical scenarios are twofold and are both induced by fast rotation: first, rotational mixing deeply affects the chemical composition 
of the material ejected by the massive stars; second, rotation significantly enhances the mass lost by stellar winds. The mass loss rates are increased mainly because the mixing process is so strong that the surface metallicity is enhanced by several orders of magnitude. This leads to strong radiative winds during the evolution in the red part of the HR diagram. The strongest mass loss occurs at the very end of the core He-burning phase. The proposed scenario may allow very massive stars to avoid the pair instability.

We show that material ejected by rotating models has chemical compositions that show close similarities to the peculiar abundance pattern observed at the surface of CEMP stars. We explored the three possibilities of CEMP stars made of: 1) massive star wind material; 2) total massive star ejecta (wind plus supernova ejecta); 3) and material from E-AGB star envelopes. Interestingly, from the models computed here, one can order these three possibilities according to the degree of richness in CNO processed material. From the richest to the poorest, one has the wind material, the E-AGB envelope, and the total ejecta of massive stars. The imprints on the abundance pattern of CEMP stars are thus not the same, depending on which source is involved. There is good hope that in the future, it will be possible to distinguish them.

Other interesting questions will be explored in the future with these rotating metal-poor models. Among them let us briefly mention:

- What is the enrichment in new synthesized helium by the first stellar generations? This is a fundamental question already asked long ago by Hoyle \& Tayler (1964). A precise knowledge of the helium enrichment caused by the first massive stars (Carr et al. 1984; Marigo et al. 2003) is important in order to correctly deduce the value of the cosmological helium from the observed abundance of helium in low metallicity regions (see e.g. Salvaterra \& Ferrara 2003). Production of helium by the first massive stars may also affect the initial helium content of stars in globular clusters. If the initial helium content of stars in globular clusters is increased by 0.02 in mass fraction, the stellar models will provide ages for the globular clusters that are lower by roughly $15 \%$, i.e., 2 Gyr starting from an age of $13 \mathrm{Gyr}$ (Shi 1995; see also the interesting discussion in Marigo et al. 2003). In the case of our rotating $60 M_{\odot}$ at $Z=10^{-8}, 22 \%$ of the initial mass is ejected in the form of new synthesised helium by stellar winds. Thus the models presented here will certainly lead to new views on the question of the helium enrichment at very low metallicity, provided of course, they are representative of the evolution of the majority of massive stars at very low $Z$.

- What are the sources of primary nitrogen in very metalpoor halo stars? Primary ${ }^{14} \mathrm{~N}$ is produced in large quantities in our rotating models. In our previous work on this subject (Meynet \& Maeder 2002), we discussed the yields from stellar models at $Z=10^{-5}$ with $v_{\text {ini }}=300 \mathrm{~km} \mathrm{~s}^{-1}$. Such an initial velocity corresponds to a ratio $v_{\text {ini }} / v_{\text {crit }}$ of only 0.25 . This value is lower than the value of $\sim 0.35$ reached by solar metallicity models with $v_{\text {ini }}=300 \mathrm{~km} \mathrm{~s}^{-1}$. With such a low initial ratio of $v_{\text {ini }} / v_{\text {crit }}$, we found that the main sources of primary nitrogen were intermediate mass stars with initial masses around about $3 M_{\odot}$. However, as already shown in Meynet \& Maeder (2002), the yield in ${ }^{14} \mathrm{~N}$ increases rapidly when the initial velocity increases. As a numerical example, the yield in primary nitrogen for the $Z=10^{-5}, 60 M_{\odot}$ with $v_{\text {ini }} / v_{\text {crit }}$ equal to 0.25 was $7 \times$ $10^{-4} M_{\odot}$, while the corresponding model with $v_{\text {ini }} / v_{\text {crit }}$ equal to 0.65 produces a yield of $\sim 2 \times 10^{-1}$; i.e., it increased by a factor of nearly 300 ! Interestingly, these high yields of primary ${ }^{14} \mathrm{~N}$ from short-lived massive stars seem to be required for explaining the high $\mathrm{N} / \mathrm{O}$ ratio observed in metal-poor halo stars (Chiappini et al. 2005). Note that massive intermediate mass stars, whose lifetimes would be only an order of magnitude higher than those of the most massive stars, could also be invoked to explain the high N/O ratio observed in very metal-poor stars. The agemetallicity relation is not precise enough to allow us to distinguish between the two.

- How does rotation affect the yields of extremely metal-poor stars? Other elements such as ${ }^{13} \mathrm{C},{ }^{17} \mathrm{O},{ }^{18} \mathrm{O}$, and ${ }^{22} \mathrm{Ne}$ are also produced in much greater quantities in the rotating models. Computations are now in progress for extending the range of initial parameters explored and to study the impact of such models on the production of these isotopes, as well as on s-process elements.

We think that the fact that stars rotate, and may even rotate fast especially at low metallicity, has to be taken into account for obtaining more realistic models of the extremely metal-poor stars that formed in the early life of the Universe.

Acknowledgements. The authors are grateful to Dr. Joli Adams for the careful language editing of the manuscript.

\section{References}

Abel, T., Bryan, G. L., \& Norman, M. L. 2002, Science, 295, 93

Angulo, C., Arnould, M., Rayet, M., et al. 1999, Nucl. Phys. A, 656, 3

Aoki, W., Norris, J. E., Ryan, S. G., et al. 2004, ApJ, 608, 971

Argast, D., Samland, M., Gerhard, O. E., \& Thielemann, F.-K. 2000, A\&A, 356, 873

Argast, D., Samland, M., Thielemann, F.-K., \& Gerhard, O. E. 2002, A\&A, 388, 842

Arnett, D. 1991, in Frontiers of Stellar Evolution, ed. D. A. Lambert, ASP Conf. Ser., 20, 385

Asplund, M., Grevesse, N., \& Sauval, J. A. 2005, in Cosmic Abundances as Records of Stellar Evolution and Nucleosynthesis, ed. F. N. Bash, \& T. G. Barnes, ASP Conf. Ser., in press [arXiv: astro-ph/0410214 v2]

Baraffe, I., El Eid, M. F., \& Prantzos, N. 1992, A\&A, 258, 357

Barkat, Z., Rakavy, G., \& Sack, N. 1967, Phys. Rev. Lett., 18, 379

Beers, T. C. 1999, in The Third Stromlo Symposium: The Galactic Halo, ed. B. K. Gibson, T. S. Axelrod, \& M. E. Putman, ASP Conf. Ser., 165, 202

Beers, T. C., Preston, G. W., \& Shectman, S. A. 1992, AJ, 103, 1987

Bessell, M. S., Christlieb, N., \& Gustafsson, B. 2004, ApJ, 612, L61

Bond, J. R., Arnett, W. D., \& Carr, B. J. 1984, ApJ, 280, 825

Carr, B. J., Bond, J. R., \& Arnett, W. D. 1984, ApJ, 277, 445

Cayrel, R., Depagne, E., Spite, M., et al. 2004, A\&A, 416, 1117

Chiappini, C., Matteucci, F., \& Ballero, S. K. 2005, A\&A, submitted 
Christlieb, N. 2003, Rev. Mod. Astron., 16, 191

Christlieb, N., Bessell, M. S., Beers, T. C., et al. 2002, Nature, 419, L904

Christlieb, N., Gustafsson, B., Korn, A. J., et al. 2004, ApJ, 603, 708

Collins, G. W., \& Sonneborn, G. H. 1977, ApJS, 34, 41

Depagne, E., Hill, V., Spite, M., et al. 2002, A\&A, 390, 187

Frebel, A., Aoki, W., Christlieb, N., et al. 2005, Nature, in press [arXiv: astro-ph/0503021]

Heger, A., \& Langer, N. 1998, A\&A, 334, 210

Heger, A., \& Langer, N. 2000, ApJ, 544, 1016

Heger, A., \& Woosley, S. E. 2002, ApJ, 567, 532

Hirschi, R., Meynet, G., \& Maeder, A. 2004, A\&A, 425, 649

Hoyle, F., \& Tayler, R. 1964, Nature, 203, 1108

Iglesias, C. A., \& Rogers, F. J. 1996, ApJ, 464, 943

Israelian, G., Ecuvillon, A., Rebolo, R., et al. 2004, A\&A, 421, 649

de Jager, C., Nieuwenhuijzen, H., \& van der Hucht, K. A. 1988, A\&AS, 72, 259

Kippenhahn, R., \& Thomas, H. C. 1970, A Simple Method for the Solution of the Stellar Structure Equations Including Rotation and Tidal Forces, in Stellar Rotation, ed. A. Slettebak (Gordon and Breach Science Publishers), IAU Coll., 4, 20

Kudritzki, R. P. 2002, ApJ, 577, 389

Kudritzki, R. P., \& Puls, J. 2000, ARA\&A, 38, 613

Kudritzki, R. P., Pauldrach, A. W. A., \& Puls, J. 1987, A\&A, 173, 293

Limongi, M., Chieffi, A., \& Bonifacio, P. 2003, ApJ, 594, L123

Lucatello, S., Tsangarides, S., Beers, T. C., et al. 2004, ApJ, in press [arXiv: astro-ph/0412422]

Madau, P. 2003, in A Massive Star Odyssey: From Main Sequence to Supernova, ed. K. van der Hucht, A. Herrero, \& C. Esteban, ASP, IAU Symp., 212, 687

Maeda, K., \& Nomoto, K. 2003, ApJ, 598, 1163

Maeder, A. 1987, A\&A, 178, 159

Maeder, A. 1992, A\&A, 264, 105

Maeder, A. 1999, A\&A, 347, 185 (Paper IV)

Maeder, A. 2003, A\&A, 399, 263
Maeder, A., \& Peytremann, E. 1970, A\&A, 7, 120

Maeder, A., \& Zahn, J. P. 1998, A\&A, 334, 1000

Maeder, A., \& Meynet, G. 2000, A\&A, 361, 159

Maeder, A., \& Meynet, G. 2001, A\&A, 373, 555 (Paper VII)

Maeder, A., Grebel, E. K., \& Mermilliod, J.-C. 1999, A\&A, 346, 459

Marigo, P., Chiosi, C., \& Kudritzki, R.-P. 2003, A\&A, 399, 617

Meynet, G., \& Maeder, A. 1997, A\&A, 321, 465 (Paper I)

Meynet, G., \& Maeder, A. 2000, A\&A, 361, 101 (Paper V)

Meynet, G., \& Maeder, A. 2002, A\&A, 390, 561 (Paper VIII)

Meynet, G., \& Maeder, A. 2003, A\&A, 404, 975 (Paper X)

Meynet, G., \& Maeder, A. 2005, A\&A, 429, 581 (Paper XI)

Meynet, G., Maeder, A., \& Ekström, S. 2005, in The Fate of the Most Massive Stars, ed. R. Humphreys, \& K. Stanek, ASP Conf. Ser., 332, in press [arXiv:astro-ph/0408322]

Norris, J. E., Ryan, S. G., \& Beers, T. C. 1997, ApJ, 488, 350

Norris, J. E., Ryan, S. G., \& Beers, T. C. 2001, ApJ, 561, 1034

Owocki, S. P., Cranmer, S. R., \& Gayley, K. G. 1996, ApJ, 472, L115

Pelló, R., Schaerer, D., Richard, J., Le Borgne, J.-F., \& Kneib, J.-P. 2005, IAU Symp. 225, ed. Y. Mellier, \& G. Meylan, in press [arXiv: astro-ph/0410132]

Picardi, I., Chieffi, A., Limongi, M., et al. 2004, ApJ, 609, 1035

Plez, B., \& Cohen, J. G. 2005, A\&A, 434, 1117

Salvaterra, R., \& Ferrara, A. 2003, MNRAS, 340, L17

Schaerer, D. 2002, A\&A, 382, 28

Schaerer, D. 2003, A\&A, 397, 527

Shi, X. 1995, ApJ, 446, 637

Shigeyama, T., \& Tsujimoto, T. 1998, ApJ, 507, L135

Spite, M., Cayrel, R., Plez, B., et al. 2005, A\&A, 430, 655

Suda, T., Aikawa, M., Machida, M. N., Fujimoto, M. Y., \& Iben, I. 2004, ApJ, 611, 476

Umeda, H., \& Nomoto, K. 2003, Nature, 412, 793

Vink, J. S., de Koter, A., \& Lamers, H. J. G. L. M. 2000, A\&A, 362, 295

Vink, J. S., de Koter, A., \& Lamers, H. J. G. L. M. 2001, A\&A, 369, 574 